JOURNAL OF THE

AMERICAN MATHEMATICAL SOCIETY

Volume 11, Number 2, April 1998, Pages 451-484

S 0894-0347(98)00260-4

\title{
GEOMETRIC REALIZATION OF WHITTAKER FUNCTIONS AND THE LANGLANDS CONJECTURE
}

\author{
E. FRENKEL, D. GAITSGORY, D. KAZHDAN, AND K. VILONEN
}

\section{INTRODUCTION}

1.1. Let $X$ be a smooth, complete, geometrically connected curve over $\mathbb{F}_{q}$. Denote by $F$ the field of rational functions on $X$, by $\mathbb{A}$ the ring of adeles of $F$, and by $\operatorname{Gal}(\bar{F} / F)$ the Galois group of $F$.

The present paper may be considered as a step towards understanding the geometric Langlands correspondence between $n$-dimensional $\ell$-adic representations of $\operatorname{Gal}(\bar{F} / F)$ and automorphic forms on the group $G L_{n}(\mathbb{A})$. We follow the approach initiated by V. Drinfeld [7], who applied the theory of $\ell$-adic sheaves to establish this correspondence in the case of $G L_{2}$.

The Langlands conjecture predicts that to any unramified irreducible $n$-dimensional representation $\sigma$ of $\operatorname{Gal}(\bar{F} / F)$, one can attach an unramified automorphic function $f_{\sigma}$ on $G L_{n}(\mathbb{A})$.

The starting point of Drinfeld's approach is the observation that an unramified automorphic function on the group $G L_{n}(\mathbb{A})$ can be viewed as a function on the set $M_{n}$ of isomorphism classes of rank $n$ bundles on the curve $X$. The set $M_{n}$ is the set of $\mathbb{F}_{q}$-points of $\mathcal{M}_{n}$, the algebraic stack of rank $n$ bundles on $X$. One may hope to construct the automorphic form associated to a Galois representation as a function corresponding to an $\ell$-adic perverse sheaf on $\mathcal{M}_{n}$. This is essentially what Drinfeld did in [7] in the case of $G L_{2}$. In abelian class field theory (the case of $G L_{1}$ ) this was done previously by P. Deligne (see [22]).

1.2. Let $M_{n}^{\prime}$ denote the set of isomorphism classes of pairs $\{L, s\}$, where $L \in M_{n}$ is a rank $n$ bundle on $X$ and $s$ is a regular non-zero section of $L$. Using a well-known construction due to Weil [34] and Jacquet-Langlands [15] for $n=2$, and Shalika [32] and Piatetski-Shapiro [30] for general $n$, one can associate a function $f_{\sigma}^{\prime}$ on $M_{n}^{\prime}$ to an unramified $n$-dimensional representation $\sigma$ of $\operatorname{Gal}(\bar{F} / F)$. The function $f_{\sigma}^{\prime}$ is obtained from the Whittaker function $W_{\sigma}$, a function canonically attached to $\sigma$. The Langlands conjecture prescribes that when $\sigma$ is geometrically irreducible, the function $f_{\sigma}^{\prime}$ should be constant along the fibers of the projection $p: M_{n}^{\prime} \rightarrow M_{n}$. In other words, conjecturally, $f_{\sigma}^{\prime}$ is the pull-back of a function $f_{\sigma}$ on $M_{n}$; the function $f_{\sigma}$ is then the automorphic function corresponding to $\sigma$. Thus, the key problem is to show that the function $f_{\sigma}^{\prime}$ is constant along the fibers of $p: M_{n}^{\prime} \rightarrow M_{n}$.

Received by the editors March 31, 1997 and, in revised form, November 26, 1997.

1991 Mathematics Subject Classification. Primary 11R39, 11F70; Secondary 14H60, 22E55.

(c)1998 American Mathematical Society 
1.3. Now let $\mathcal{M}_{n}^{\prime}$ be the moduli stack of pairs $\{L, s\}$, where $L$ is a rank $n$ bundle on $X$ and $s$ is a regular non-zero section of $L$. We have: $M_{n}=\mathcal{M}_{n}\left(\mathbb{F}_{q}\right), M_{n}^{\prime}=\mathcal{M}_{n}^{\prime}\left(\mathbb{F}_{q}\right)$. Each Galois representation $\sigma$ gives rise to an $\ell$-adic local system $E$ on $X$ of rank $n$. Drinfeld's idea, developed further by G. Laumon [22], can be interpreted as follows.

Suppose there exists an irreducible perverse sheaf $\mathcal{S}_{E}^{\prime}$ on $\mathcal{M}_{n}^{\prime}$, with the property that the function $S_{E}^{\prime}$ associated to $\mathcal{S}_{E}^{\prime}$ on $M_{n}^{\prime}=\mathcal{M}_{n}^{\prime}\left(\mathbb{F}_{q}\right)$ equals $f_{\sigma}^{\prime}$. Then showing that the function $f_{\sigma}^{\prime}$ is constant along the fibers of the projection $p: M_{n}^{\prime} \rightarrow M_{n}$ becomes a geometric problem of proving that $\mathcal{S}_{E}^{\prime}$ descends to a perverse sheaf $\mathcal{S}_{E}$ on $\mathcal{M}_{n}$.

In [7], Drinfeld constructed such a sheaf $\mathcal{S}_{E}^{\prime}$ in the case of $G L_{2}$. He started with a geometric realization of the Whittaker function as a perverse sheaf on the symmetric power of the curve $X$. Then he defined the sheaf $\mathcal{S}_{E}^{\prime}$ using a geometric version of the Weil-Jacquet-Langlands construction. Drinfeld showed that the sheaf $\mathcal{S}_{E}^{\prime}$ is locally constant along the fibers of $p$. Since the fibers of $p$ are projective spaces, hence simply-connected, this implies that $\mathcal{S}_{E}^{\prime}$ is constant along the fibers of $p$. Drinfeld's construction raised hope that one can use a similar argument in the case of $G L_{n}$.

1.4. An analogue of Drinfeld's construction in the case of $G L_{n}$ has been proposed by Laumon in [22], [25]. In order to construct $\mathcal{S}_{E}^{\prime}$ for $G L_{n}$, he defined a sheaf $\mathcal{L}_{E}$, which he considered as a geometric analogue of the Whittaker function $W_{\sigma}$. However, the function $L_{E}$ corresponding to $\mathcal{L}_{E}$ and the Whittaker function $W_{\sigma}$ are defined on different sets and their values are different; see [22] and Sect. 3.5 below. Using the sheaf $\mathcal{L}_{E}$, Laumon [25] constructed a candidate for the sheaf $\mathcal{S}_{E}^{\prime}$ on $\mathcal{M}_{n}^{\prime}$ (this construction was independently found by D. Gaitsgory, unpublished). In order to justify this construction, one has to prove that the function $S_{E}^{\prime}$ on $M_{n}^{\prime}$, corresponding to the sheaf $\mathcal{S}_{E}^{\prime}$, coincides with the function $f_{\sigma}^{\prime}$. This equality was conjectured by Laumon in [25] (Conjecture 3.2), and its proof is one of the main goals of this paper.

To prove the equality $S_{E}^{\prime}=f_{\sigma}^{\prime}$, we reduce it to a local statement (see Theorem 5.2) which we make for an arbitrary reductive group. We prove Theorem 5.2 using the Casselman-Shalika formula for the Whittaker function [5] (actually, Theorem 5.2 is equivalent to the Casselman-Shalika formula). Theorem 5.2 can be translated into a geometric statement about intersection cohomology sheaves on the affine Grassmannian (see Conjecture 7.2).

1.5. One essential difference between Laumon's approach and our approach is in interpretation of the sheaf $\mathcal{L}_{E}$ and the function $L_{E}$ associated to this sheaf. Laumon interprets the local factors of $L_{E}$ via the Springer sheaves and the KostkaFoulkes polynomials (see Remark 3.1). We interpret the local factors of $L_{E}$ via the perverse sheaves on the affine Grassmannian and the Hecke algebra (see Sect. 4.2). Our interpretation, which was inspired by [26], allows us to gain more insight into Laumon's construction. In particular, it helps to explain the apparent discrepancy between $L_{E}$ and $W_{\sigma}$ : it turns out that $L_{E}$ is related to $W_{\sigma}$ by a Fourier transform. Using this result, we demonstrate that the output of the two constructions $-S_{E}^{\prime}$ and $f_{\sigma}^{\prime}$ - coincide.

1.6. Let us now briefly describe the contents of the paper:

In Sect. 2 we review some background material concerning the Langlands conjecture and the classical construction of the function $f_{\sigma}^{\prime}$ together with its geometric interpretation. We follow closely Sect. 1 of [22]. 
In Sect. 3 we describe the construction of the sheaf $\mathcal{S}_{E}^{\prime}$ on $\mathcal{M}_{n}^{\prime}$ and the function $S_{E}^{\prime}$ on $M_{n}^{\prime}$. We state the main conjecture (Conjecture 3.1 ) about the geometric Langlands correspondence for $G L_{n}$ and our main result (Theorem 3.1).

In Sect. 4 we give an adelic interpretation of the construction of the function $S_{E}^{\prime}$ and reduce Theorem 3.1 to a local statement, Proposition 4.2.

In Sect. 5 we prove Theorem 5.2 for an arbitrary reductive group and derive from it Proposition 4.2.

In Sect. 6 we interpret the results of Sect. 5 from the point of view of the spherical functions.

In Sect. 7 we give a geometric interpretation of Theorem 5.2 and discuss a possible generalization of Laumon's construction to other groups.

1.7. In this paper we work with algebraic stacks in the smooth topology in the sense of [24]. All stacks that we consider have locally the form $y / G$ where $y$ is a scheme and $G$ is an algebraic group acting on it. We will use the following notion of perverse sheaves on such algebraic stacks: for $\mathcal{V}=y / G$, a perverse sheaf on $\mathcal{V}$ is just a $G$-equivariant perverse sheaf on $y$, appropriately shifted.

Throughout this paper, for an $\mathbb{F}_{q}$-scheme (resp., for an $\mathbb{F}_{q}$-stack) $\mathcal{V}$ and for an algebra $R$ over $\mathbb{F}_{q}, \mathcal{V}(R)$ will denote the set of $R$-points of $\mathcal{V}$ (resp., the set of isomorphism classes of $R$-points of $\mathcal{V}$ ). In most cases, schemes and stacks are denoted by script letters and their sets of $\mathbb{F}_{q}$-points are denoted by the corresponding italic letters (e.g., $v$ and $V$ ). We use the same notation for a morphism of stacks and for the corresponding map of sets.

If $\mathcal{S}$ is a sheaf or a complex of sheaves on a stack $\mathcal{V}$, then the corresponding set $V$ is endowed with a function "alternating sum of traces of the Frobenius" (as in [6]) which we denote by the corresponding italic letter $S$ (we assume that a square root of $q$ in $\overline{\mathbb{Q}}_{\ell}$ is fixed throughout the paper).

If $\mathcal{V}$ is a stack over $\mathbb{F}_{q}$, the set $V$ is endowed with a canonical measure $\mu$, which in the case when $\mathcal{V}$ is a scheme has the property $\mu(v)=1, \forall v \in V$. For example, if $\mathcal{G}$ is a group, $\mu\left((p t / \mathcal{G})\left(\mathbb{F}_{q}\right)\right)=|G|^{-1}$.

\section{Background and the Shalika-Piatetski-Shapiro construction}

2.1. Langlands conjecture. Let $k=\mathbb{F}_{q}$ be a finite field, and let $X$ be a smooth complete geometrically connected curve over $k$. Denote by $F$ the field of rational functions on $X$. For each closed point $x$ of $X$, denote by $\mathcal{K}_{x}$ the completion of $F$ at $x$, by $\mathcal{O}_{x}$ the ring of integers of $\mathcal{K}_{x}$, and by $\pi_{x}$ a generator of the maximal ideal of $\mathcal{O}_{x}$. Let $k_{x}$ be the residue field $\mathcal{O}_{x} / \pi_{x} \mathcal{O}_{x}$, and let $q_{x}=q^{\operatorname{deg} x}$ be its cardinality. We denote by $\mathbb{A}=\prod_{x \in|X|}^{\prime} \mathcal{K}_{x}$ the ring of adeles of $F$ and by $\mathcal{O}=\prod_{x \in|X|}^{\prime} \mathcal{O}_{x}$ its maximal compact subring.

Consider the set $\mathfrak{G}_{n}$ of unramified and geometrically irreducible $\ell$-adic representations of the Galois group $\operatorname{Gal}(\bar{F} / F)$ in $G L_{n}\left(\overline{\mathbb{Q}}_{\ell}\right)$, where $\ell$ is relatively prime to $q$, as in [22], (1.1).

Let $\mathfrak{A}_{n}$ be the set of cuspidal unramified automorphic functions on the group $G L_{n}(\mathbb{A})$ - these are cuspidal functions on the set $G L_{n}(F) \backslash G L_{n}(\mathbb{A}) / G L_{n}(\mathcal{O})$, which are eigenfunctions of the Hecke operators. Recall that for each $x \in|X|$ and $i=$ $1, \ldots, n$, one defines the Hecke operator $T_{x}^{i}$ by the formula:

$$
\left(T_{x}^{i} \cdot f\right)(g)=\int_{M_{n}^{i}\left(\mathcal{O}_{x}\right)} f(g h) d h,
$$


where

$$
M_{n}^{i}\left(\mathcal{O}_{x}\right)=G L_{n}\left(\mathcal{O}_{x}\right) \cdot D_{x}^{i} \cdot G L_{n}\left(\mathcal{O}_{x}\right) \subset G L_{n}\left(\mathcal{K}_{x}\right) \subset G L_{n}(\mathbb{A}) ;
$$

here $D_{x}^{i}$ is the diagonal matrix whose first $i$ entries equal $\pi_{x}$, and the remaining $n-i$ entries equal 1 , and $d h$ stands for the Haar measure on $G L_{n}\left(\mathcal{K}_{x}\right)$ normalized so that $G L_{n}\left(\mathcal{O}_{x}\right)$ has measure 1 .

Let $B$ be the Borel subgroup of upper triangular matrices and $T N$ its standard Levi decomposition. Cuspidality condition means that for each proper parabolic subgroup of $G L_{n}$, whose unipotent radical $V$ is contained in the upper unipotent subgroup $N$,

$$
\int_{V(F) \backslash V(\mathbb{A})} f(v g) d v=0, \quad \forall g \in G L_{n}(\mathbb{A}) .
$$

Conjecture 2.1. For each $\sigma \in \mathfrak{G}_{n}$, there exists a unique (up to a non-zero constant multiple) function $f_{\sigma} \in \mathfrak{A}_{n}$, such that for any $x \in|X|$

$$
T_{x}^{i} \cdot f_{\sigma}=q_{x}^{-i(i-1) / 2} \operatorname{Tr}\left(\Lambda^{i} \sigma\left(\operatorname{Fr}_{x}\right)\right) f_{\sigma}, \quad i=1, \ldots, n,
$$

where $T_{x}^{i}$ is the ith Hecke operator, and $\operatorname{Fr}_{x} \in \operatorname{Gal}(\bar{F} / F)$ is the geometric Frobenius element.

Let $P_{1} \subset G L_{n}$ be the subgroup

$$
\left\{\left(\begin{array}{cc}
g & h \\
0 & 1
\end{array}\right) \mid g \in G L_{n-1}\right\} .
$$

Following Shalika and Piatetski-Shapiro, we construct a function $f_{\sigma}^{\prime}$ on the doublequotient

$$
P_{1}(F) \backslash G L_{n}(\mathbb{A}) / G L_{n}(\mathcal{O}),
$$

which is cuspidal and which satisfies the Hecke eigenfunction property:

$$
T_{x}^{i} \cdot f_{\sigma}^{\prime}=q_{x}^{-i(i-1) / 2} \operatorname{Tr}\left(\Lambda^{i} \sigma\left(\operatorname{Fr}_{x}\right)\right) f_{\sigma}^{\prime}, \quad i=1, \ldots, n, \forall x \in|X| .
$$

The first step in the construction of $f_{\sigma}^{\prime}$ is the construction of the Whittaker function.

2.2. Whittaker functions. Introduce the following notation: for a homomorphism Spec $R \rightarrow X$ and an $\mathcal{O}_{X}$-module $M$ we denote by $M_{R}$ the $R$-module of sections of the pull-back of $M$ to $\operatorname{Spec} R$. Denote by $\Omega$ the canonical bundle over $X$. Let $G L_{n}^{J}(R)$ be the group of invertible $n \times n$ matrices $A=\left(A_{i j}\right)_{0 \leq i, j \leq n-1}$, where $A_{i j} \in \Omega_{R}^{j-i}$. The group $G L_{n}^{J}(R)$ is locally (for the Zariski topology) isomorphic to the corresponding non-twisted group $G L_{n}(R)$. To establish such an isomorphism, one has to choose a non-vanishing regular section $\delta$ of $\Omega$, so the isomorphism is not canonical.

It is easy to see that $G L_{n}^{J}(R)$ is the group of $R$-points of a group scheme over $X$, but in this paper we will not use this fact.

We denote by $N^{J}(R), T^{J}(R), P_{1}^{J}(R)$, etc., the corresponding subgroups of $G L_{n}^{J}(R)$.

The twisted forms $G L_{n}^{J}(R)$ have the following advantage. Let $u_{i, i+1}$ be the $i$ th component of the image of $u \in N^{J}(R)$ in $N^{J}(R) /\left[N^{J}(R), N^{J}(R)\right]$ corresponding to the $(i, i+1)$ entry of $u$. Then $u_{i, i+1} \in \Omega_{R}$. 
Let us fix once and for all a non-trivial additive character $\psi: k \rightarrow \overline{\mathbb{Q}}_{\ell}^{\times}$. We define the character $\Psi_{x}$ of $N^{J}\left(\mathcal{K}_{x}\right)$ by the formula

$$
\Psi_{x}(u)=\prod_{i=1}^{n-1} \psi\left(\operatorname{Tr}_{k_{x} / k}\left(\operatorname{Res}_{x} u_{i, i+1}\right)\right)
$$

and the character $\Psi$ of $N^{J}(\mathbb{A})$ by

$$
\Psi\left(\left(u_{x}\right)\right)=\prod_{x \in|X|} \Psi_{x}\left(u_{x}\right) .
$$

It follows that $\Psi(u)=1$ if $u \in N^{J}(\mathcal{O})$ or $u \in N^{J}(F)$.

For each $x \in|X|$ consider the group $G L_{n}^{J}\left(\mathcal{K}_{x}\right)$. Let $\gamma$ be a semi-simple conjugacy class in $G L_{n}\left(\overline{\mathbb{Q}}_{\ell}\right)$. The following result is due to Shintani [33], and Casselman and Shalika [5].

Theorem 2.1. (1) There exists a unique function $W_{\gamma, x}$ on $G L_{n}^{J}\left(\mathcal{K}_{x}\right)$ that satisfies the following properties:

- $W_{\gamma, x}(g h)=W_{\gamma, x}(g), \forall h \in G L_{n}^{J}\left(\mathcal{O}_{x}\right), W_{\gamma, x}(1)=1$;

- $W_{\gamma, x}(u g)=\Psi_{x}(u) W_{\gamma, x}(g), \forall u \in N^{J}\left(\mathcal{K}_{x}\right)$;

- $W_{\gamma, x}$ is an eigenfunction with respect to the local Hecke-operators $T_{x}^{i}, i=$ $1, \ldots, n$ :

$$
T_{x}^{i} \cdot W_{\gamma, x}=q_{x}^{-i(i-1) / 2} \operatorname{Tr}\left(\Lambda^{i}(\gamma)\right) W_{\gamma, x} .
$$

(2) The function $W_{\gamma, x}$ is given by the following formulas.

For $\lambda=\left(\lambda_{1}, \ldots, \lambda_{n}\right) \in P_{n}^{+}$, the set of dominant weights of $G L_{n}$ (i.e., such that $\left.\lambda_{1} \geq \lambda_{2} \geq \ldots \geq \lambda_{n}\right)$,

$$
W_{\gamma, x}\left(\operatorname{diag}\left(\pi_{x}^{\lambda_{1}}, \ldots, \pi_{x}^{\lambda_{n}}\right)\right)=q_{x}^{n(\lambda)} \operatorname{Tr}(\gamma, V(\lambda)),
$$

where $V(\lambda)$ is the irreducible representation of $G L_{n}\left(\overline{\mathbb{Q}}_{\ell}\right)$ of highest weight $\lambda$, and $n(\lambda)=\sum_{i=1}^{n}(i-1) \lambda_{i}$.

For $\lambda \in \mathbb{Z}^{n}-P_{n}^{+}, W_{\gamma, x}\left(\operatorname{diag}\left(\pi_{x}^{\lambda_{1}}, \ldots, \pi_{x}^{\lambda_{n}}\right)\right)=0$.

There is a bijection between the weight lattice of $G L_{n}$ and the double quotient $N^{J}\left(\mathcal{K}_{x}\right) \backslash G L_{n}^{J}\left(\mathcal{K}_{x}\right) / G L_{n}^{J}\left(\mathcal{O}_{x}\right)$, which maps $\left(\lambda_{1}, \ldots, \lambda_{n}\right)$ to the double coset of $\operatorname{diag}\left(\pi_{x}^{\lambda_{1}}, \ldots, \pi_{x}^{\lambda_{n}}\right)$. This explains the fact that $W_{\gamma, x}$ is uniquely determined by its values at the points $\operatorname{diag}\left(\pi_{x}^{\lambda_{1}}, \ldots, \pi_{x}^{\lambda_{n}}\right)$.

Remark 2.1. The uniqueness of the Whittaker function is connected with the fact that an irreducible smooth representation of a reductive group $G$ over a local nonarchimedian field has at most one Whittaker model; see [9].

There is an explicit formula for the Whittaker function associated to an arbitrary reductive group, due to Casselman and Shalika [5], which we will use in Sect. 5.

Now we attach to $\sigma \in \mathfrak{A}_{n}$ the global Whittaker function $W_{\sigma}$ on $G L_{n}^{J}(\mathbb{A})$ by the formula

$$
W_{\sigma}\left(\left(g_{x}\right)\right)=\prod_{x \in|X|} W_{\sigma\left(\operatorname{Fr}_{x}\right), x}\left(g_{x}\right) .
$$

It satisfies:

- $W_{\sigma}(g h)=W_{\sigma}(g), \forall h \in G L_{n}^{J}(\mathcal{O}), W_{\sigma}(1)=1$;

- $W_{\sigma}(u g)=\Psi(u) W_{\sigma}(g), \forall u \in N^{J}(\mathbb{A})$; in particular, $W_{\sigma}$ is left invariant with respect to $N^{J}(F)$; 
- For all $i=1, \ldots, n$ and $x \in|X|$ we have:

$$
T_{x}^{i} \cdot W_{\sigma}=q_{x}^{-i(i-1) / 2} \operatorname{Tr}\left(\Lambda^{i} \sigma\left(F r_{x}\right)\right) W_{\sigma} .
$$

2.3. The construction of $f_{\sigma}^{\prime}$. Let $C^{\infty}\left(G L_{n}^{J}(\mathbb{A})\right)_{\Psi}^{N^{J}(\mathbb{A})}$ be the space of $\overline{\mathbb{Q}}_{\ell}$-valued smooth (see, e.g., [3]) functions $f$ on $G L_{n}^{J}(\mathbb{A})$, such that $f(u g)=\Psi(u) f(g), \forall u \in$ $N^{J}(\mathbb{A})$; we call such functions $\left(N^{J}(A), \Psi\right)$-equivariant. Let $C^{\infty}\left(G L_{n}^{J}(\mathbb{A})\right)_{\text {cusp }}^{P_{1}^{J}(F)}$ be the space of smooth functions $f$ on $G L_{n}^{J}(\mathbb{A})$, which satisfy $f(p g)=f(g), \forall p \in P_{1}^{J}(F)$ and are cuspidal, i.e., for each proper parabolic subgroup of $G L_{n}$, whose unipotent radical $V$ is contained in $N$,

$$
\int_{V^{J}(F) \backslash V^{J}(\mathbb{A})} f(v g) d v=0, \quad \forall g \in G L_{n}^{J}(\mathbb{A}) .
$$

The following result is the main step in constructing automorphic functions for $G L_{n}$. The existence of the subgroup $P_{1}$ plays a key role in this result, and this makes the case of $G L_{n}$ special.

Theorem 2.2. There is a canonical isomorphism

$$
\phi: C^{\infty}\left(G L_{n}^{J}(\mathbb{A})\right)_{\Psi}^{N^{J}(\mathbb{A})} \rightarrow C^{\infty}\left(G L_{n}^{J}(\mathbb{A})\right)_{\text {cusp }}^{P_{1}^{J}(F)}
$$

given by the formula

$$
(\phi(f))(g)=\sum_{y \in N_{n-1}^{J}(F) \backslash G L_{n-1}^{J}(F)} f\left(\left(\begin{array}{ll}
y & 0 \\
0 & 1
\end{array}\right) g\right) .
$$

This isomorphism commutes with the right action of $G L_{n}^{J}(\mathbb{A})$ on both spaces.

For the proof, see [32] and [30]. Note that Theorem 2.2 is not stated exactly in this form in [32] but its proof can be extracted from the proof of Theorem 5.9 there. We remark that for each $g \in G L_{n}^{J}(\mathbb{A})$ the sum above has finitely many non-zero terms.

By construction, $W_{\sigma} \in C^{\infty}\left(G L_{n}^{J}(\mathbb{A})\right)_{\Psi}^{N^{J}(\mathbb{A})}$. Let $f_{\sigma}^{\prime}=\phi\left(W_{\sigma}\right)$. The isomorphism of Theorem 2.2 clearly preserves the spaces of right $G L_{n}^{J}(\mathcal{O})$-invariant functions and commutes with the action of the Hecke operators on them. Therefore $f_{\sigma}^{\prime}$ is right $G L_{n}^{J}(\mathcal{O})$-invariant and satisfies formula (2.2), i.e., it is an eigenfunction of the Hecke operators with the same eigenvalues as those prescribed by the Langlands conjecture. Furthermore, uniqueness of the Whittaker function $W_{\sigma}$ implies that the function $f_{\sigma}^{\prime}$ is the unique function on $G L_{n}^{J}(\mathbb{A})$ satisfying the above properties (up to a non-zero constant multiple). Thus, the Langlands Conjecture 2.1 is equivalent to

Conjecture 2.2. For each $\sigma \in \mathfrak{G}_{n}$, the function $f_{\sigma}^{\prime}$ is left $G L_{n}^{J}(F)$-invariant.

Remark 2.2. There is an approach to proving this conjecture using analytic properties of the $L$-function of the Galois representation $\sigma$ (see [15], [16], [21]), which will not be discussed here.

2.4. Interpretation in terms of vector bundles. We begin by fixing notation. Recall that $B$ is the Borel subgroup in $G L_{n}$ (consisting of upper triangular matrices) and $T \subset B$ is the maximal torus (consisting of diagonal matrices). Let $P$ be the maximal parabolic subgroup of $G L_{n}$ containing the subgroup $P_{1}$ of $G L_{n}$ defined by formula (2.1). 
Denote $T^{J}\left(\mathcal{K}_{x}\right)^{+}=T^{J}\left(\mathcal{K}_{x}\right) \cap \operatorname{Diag}\left(\mathcal{O}_{x}\right)$, where $\operatorname{Diag}\left(\mathcal{O}_{x}\right)$ is the set of diagonal $n \times n$ matrices with coefficients in $\mathcal{O}_{x}, B^{J}\left(\mathcal{K}_{x}\right)^{+}=N^{J}\left(\mathcal{K}_{x}\right) T^{J}\left(\mathcal{K}_{x}\right)^{+}$and

$$
P^{J}\left(\mathcal{K}_{x}\right)^{+}=\left\{\left(\begin{array}{ll}
a & b \\
0 & c
\end{array}\right)\left|a \in G L_{n-1}^{J}\left(\mathcal{K}_{x}\right), c \in \mathcal{K}_{x}^{\times},\right| c \mid \leq 1\right\} .
$$

Let $B^{J}(\mathbb{A})^{+}=\prod_{x \in|X|}^{\prime} B^{J}\left(\mathcal{K}_{x}\right)^{+}$and $P^{J}(\mathbb{A})^{+}=\prod_{x \in|X|}^{\prime} P^{J}\left(\mathcal{K}_{x}\right)^{+}$.

Denote by $Q$ the double quotient $N^{J}(F) \backslash B^{J}(\mathbb{A})^{+} / B^{J}(\mathcal{O})$. Note that since

$$
B^{J}(\mathbb{A}) / B^{J}(\mathcal{O}) \simeq G L_{n}^{J}(\mathbb{A}) / G L_{n}^{J}(\mathcal{O})
$$

$Q$ is naturally a subset of $N^{J}(F) \backslash G L_{n}^{J}(\mathbb{A}) / G L_{n}^{J}(\mathcal{O})$.

Let $M_{n}^{\prime}$ denote the double quotient $P_{1}^{J}(F) \backslash P^{J}(\mathbb{A})^{+} / P^{J}(\mathcal{O})$. Note that since

$$
P^{J}(\mathbb{A}) / P^{J}(\mathcal{O}) \simeq G L_{n}^{J}(\mathbb{A}) / G L_{n}^{J}(\mathcal{O}),
$$

$M_{n}^{\prime}$ is naturally a subset of $P_{1}^{J}(F) \backslash G L_{n}^{J}(\mathbb{A}) / G L_{n}^{J}(\mathcal{O})$.

Finally, set $M_{n}=G L_{n}^{J}(F) \backslash G L_{n}^{J}(\mathbb{A}) / G L_{n}^{J}(\mathcal{O})$.

Let us denote by $\nu$ and $p$ the obvious projections $Q \rightarrow M_{n}^{\prime}$ and $M_{n}^{\prime} \rightarrow M_{n}$, respectively.

Lemma 2.1. (1) There is a canonical bijection between the set $Q$ and the set of isomorphism classes of the following data: $\left\{L,\left(F_{i}\right),\left(s_{i}\right)\right\}$, where $L$ is a rank $n$ vector bundle over $X, 0=F_{n} \subset F_{n-1} \subset \ldots \subset F_{1} \subset F_{0}=L$ is a full flag of subbundles in $L$, and $s_{i}: \Omega^{i} \rightarrow L_{i}=F_{i} / F_{i+1}$ is a non-zero $\mathcal{O}_{X}$-module homomorphism.

(2) There is a canonical bijection between the set $M_{n}^{\prime}$ and the set of isomorphism classes of pairs $\{L, s\}$, where $L$ is a rank $n$ vector bundle over $X$, and $s: \Omega^{n-1} \rightarrow L$ is a non-zero $\mathcal{O}_{X}$-module homomorphism.

The natural projection $\nu: Q \rightarrow M_{n}^{\prime}$ sends $\left\{L,\left(F_{i}\right),\left(s_{i}\right)\right\} \in Q$ to $\left\{L, s_{n-1}\right\} \in M_{n}^{\prime}$.

(3) There is a canonical bijection between the set $M_{n}$ and the set of isomorphism classes of rank $n$ vector bundles over $X$.

The natural map $p: M_{n}^{\prime} \rightarrow M_{n}$ corresponds to forgetting the section $s$.

Proof. Recall that for a morphism $\operatorname{Spec} R \rightarrow X$ and an $\mathcal{O}_{X}$-module $M$ we denote by $M_{R}$ the space of sections of the pull-back of $M$ to $\operatorname{Spec} R$. Denote by $J^{0}$ the vector bundle $\bigoplus_{i=0}^{n-1} \Omega^{i}$.

Let $B$ un be the set of data $\left\{L, \varphi_{\text {gen }}, \varphi_{x}\right\}$, where $L$ is a rank $n$ bundle on $X$, and $\varphi_{\text {gen }}: J_{F}^{0} \rightarrow L_{F}$ and $\varphi_{x}: J_{\mathcal{O}_{x}}^{0} \rightarrow L_{\mathcal{O}_{x}}, \forall x \in|X|$, are isomorphisms (generic and local "trivializations", respectively). We construct a map $b: B u n \rightarrow G L_{n}^{J}(\mathbb{A})$ as follows. After the identifications of $J_{F}^{0} \otimes_{F} \mathcal{K}_{x} \simeq J_{\mathcal{O}_{x}}^{0} \otimes_{\mathcal{O}_{x}} \mathcal{K}_{x}$ with $J_{\mathcal{K}_{x}}^{0}$, and of $L_{F} \otimes_{F} \mathcal{K}_{x} \simeq L_{\mathcal{O}_{x}} \otimes_{\mathcal{O}_{x}} \mathcal{K}_{x}$ with $L_{\mathcal{K}_{x}}, \varphi_{x}$ and $\varphi_{\text {gen }}$ give rise to homomorphisms $J_{\mathcal{K}_{x}}^{0} \rightarrow L_{\mathcal{K}_{x}}$ which we denote by the same characters. Let $\mu_{x}=\left(\varphi_{x}\right)^{-1} \varphi_{\text {gen }}$ be the corresponding automorphism of $J_{\mathcal{K}_{x}}^{0}$.

To represent the element $\mu_{x}$ by an $n \times n$ matrix $g_{x}=\left(g_{x, i j}\right)$ of the form given in Sect. 2.2, we set $g_{x, i j}$ to be equal to the element of $\Omega_{\mathcal{K}_{x}}^{j-i}$ corresponding to the map $\Omega_{\mathcal{K}_{x}}^{i} \rightarrow J_{\mathcal{K}_{x}}^{0} \stackrel{\mu_{x}}{\longrightarrow} J_{\mathcal{K}_{x}}^{0} \rightarrow \Omega_{\mathcal{K}_{x}}^{j}$. Thus, $g_{x}$ is the transpose ${ }^{1}$ of the matrix representing the action of $\mu_{x}$ on $J_{\mathcal{K}_{x}}^{0}$. The map $b$ sends $\left\{L, \varphi_{\text {gen }}, \varphi_{x}\right\}$ to $\left(g_{x}\right)_{x \in|X|} \in G L_{n}^{J}(\mathbb{A})$. It is easy to see that this map is a bijection.

Now to prove part (1) of the lemma, let us observe that given a triple $\left\{L,\left(F_{i}\right)\right.$, $\left.\left(s_{i}\right)\right\}$, we can choose $\varphi_{\text {gen }}$ and $\varphi_{x}$ 's in such a way that for each $j=0, \ldots, n-1$,

\footnotetext{
${ }^{1}$ Taking the transpose has some advantages, in particular, it agrees with the conventions adopted in [22].
} 
they map $\bigoplus_{i=j}^{n-1} \Omega_{R}^{i}$ to $F_{j, R} \subset L_{R}$ and the associated maps $\Omega_{R}^{j} \rightarrow F_{j, R} / F_{j+1, R}$ coincide with restrictions of $s_{j}$ to $\operatorname{Spec} R$ (here $R=F$ or $\mathcal{O}_{x}$ ). With such a choice, $g_{x} \in B^{J}\left(\mathcal{K}_{x}\right)^{+}, \forall x \in|X|$, and the arbitrariness in the choice of $\varphi_{\text {gen }}$ (resp., $\varphi_{x}$ ) corresponds to left (resp., right) multiplication of $\left(g_{x}\right)_{x \in|X|}$ by elements of $N^{J}(F)$ (resp., $\left.B^{J}\left(\mathcal{O}_{x}\right)\right)$.

This proves part (1) of the lemma. The proof of parts (2) and (3) is similar.

Note that the function $W_{\sigma}$ (resp., $f_{\sigma}^{\prime}$ ), which is defined on the set

$$
N^{J}(F) \backslash G L_{n}^{J}(\mathbb{A}) / G L_{n}^{J}(\mathcal{O})
$$

(resp., $\left.P_{1}^{J}(F) \backslash G L_{n}^{J}(\mathbb{A}) / G L_{n}^{J}(\mathcal{O})\right)$ is uniquely determined by its restriction to the subset $Q$ (resp., $M_{n}^{\prime}$ ), since it is an eigenfunction of the Hecke operators $T_{x}^{n}$.

Now $f_{\sigma}^{\prime}$ is a function on $P_{1}^{J}(F) \backslash G L_{n}^{J}(\mathbb{A}) / G L_{n}^{J}(\mathcal{O})$. Its restriction to $M_{n}^{\prime}$, which we also denote by $f_{\sigma}^{\prime}$, equals, by definition, $\nu_{!}\left(W_{\sigma}\right)$, where $\nu_{\text {! denotes the operation }}$ of summing up a function along the fibers of the map $\nu$ (note that these fibers are finite). Conjecture 2.2 can now be stated in the following way.

Conjecture 2.3. The function $f_{\sigma}^{\prime}$ is constant along the fibers of the map $p: M_{n}^{\prime} \rightarrow$ $M_{n}$.

In the next section we discuss a geometric version of this conjecture.

\section{Conjectural geOmetric CONSTRUCtion of an AUtomorphic Sheaf}

3.1. Definitions of stacks. Let $\mathcal{M}_{n}$ be the moduli stack of rank $n$ bundles on $X$. Recall that for an $\mathbb{F}_{q}$-scheme $S, \operatorname{Hom}\left(S, \mathcal{M}_{n}\right)$ is the groupoid whose objects are rank $n$ bundles on $X \times S$ and morphisms are isomorphisms of such bundles. Let $\mathcal{M}_{n}^{\prime}$ be the moduli stack of pairs $\{L, s\}$, where $L$ is a rank $n$ bundle on $X$ and $s: \Omega^{n-1} \rightarrow \mathcal{M}_{n}^{\prime}$ is an embedding of $\mathcal{O}_{X}$-modules. More precisely, $\operatorname{Hom}\left(S, \mathcal{N}_{n}^{\prime}\right)$ is the groupoid whose objects are pairs $\left\{L_{S}, s_{S}\right\}$, where $L_{S} \in \operatorname{Ob} \operatorname{Hom}\left(S, \mathcal{M}_{n}\right)$ and $s_{S}: \Omega_{X}^{n-1} \otimes \mathcal{O}_{S} \rightarrow L_{S}$ is an embedding, such that the quotient $L_{S} / \operatorname{Im} s_{S}$ is $S_{-}$ flat; morphisms are isomorphisms of such pairs which make the natural diagram commutative.

The set $M_{n}$ (resp. $M_{n}^{\prime}$ ) can be identified with the set of $\mathbb{F}_{q}$-points of $\mathcal{M}_{n}$ (resp. $\mathcal{M}_{n}^{\prime}$ ). As was explained in the introduction, we expect that $f_{\sigma}^{\prime}$ is the function attached to a complex of $\ell$-adic sheaves $\mathcal{S}_{E}^{\prime}$ on $\mathcal{M}_{n}^{\prime}$. In this section we present the construction of a candidate for the complex $\mathcal{S}_{E}^{\prime}$ following Laumon [25]. At the level of $\mathbb{F}_{q}$-points, this construction is actually different from the construction of $f_{\sigma}^{\prime}$ given in Sect. 2.3.

The reason is the following. It is easy to define a "naive" stack $Q$ classifying triples $\left\{L,\left(F_{i}\right),\left(s_{i}\right)\right\}$ (as in Lemma 2.1) with $\mathcal{Q}\left(\mathbb{F}_{q}\right)=Q$ and a morphism $\mathcal{Q} \rightarrow \mathcal{M}_{n}^{\prime}$ corresponding to the map of sets $\nu: Q \rightarrow M_{n}^{\prime}$. But this $Q$ is a disjoint union of connected components labeled by the $n$-tuples $\left(d_{0}, \ldots, d_{n-1}\right)$, where $d_{i}$ is the degree of the divisor of zeros of the map $s_{i}: \Omega^{i} \rightarrow F_{i} / F_{i+1}$. On the other hand, the stack $\mathcal{M}_{n}^{\prime}$ is a disjoint union of connected components corresponding to the degree of the divisor of zeros of $s: \Omega^{n-1} \rightarrow L$. Recall that under $\nu, s_{n-1}$ becomes $s$. This means that the fibers of $\nu$ are disconnected. Hence one cannot obtain an irreducible sheaf on $\mathcal{M}_{n}^{\prime}$ as the direct image of a sheaf on $\mathcal{Q}$.

In this section we replace the "naive" stack $\mathcal{Q}$ by a stack $\widetilde{Q}$, and the Whittaker function $W_{\sigma}$ by a perverse sheaf $\mathcal{F}_{E}$ on $\widetilde{\mathcal{Q}}$. The pair $\left(\widetilde{\mathcal{Q}}, \mathcal{F}_{E}\right)$ was first constructed by Laumon [25]. 
3.2. The stack $\widetilde{\mathcal{Q}}$. The algebraic stack $\widetilde{\mathcal{Q}}$ is defined as follows. For an $\mathbb{F}_{q}$-scheme $S$, $\operatorname{Hom}(S, \widetilde{Q})$ is the groupoid whose objects are quintuples $\left\{L_{S}, \mathbf{s}_{S}, J_{S},\left(J_{i, S}\right),\left(s_{i, S}\right)\right\}$, where $L_{S}$ and $J_{S}$ are rank $n$ bundles on $X \times S, \mathbf{s}_{S}: J_{S} \rightarrow L_{S}$ is an embedding of the corresponding $\mathcal{O}_{X \times S}$-modules, such that the quotient is $S$-flat, $\left(J_{i, S}\right)$ is a full flag of subbundles

$$
0=J_{n, S} \subset J_{n-1, S} \subset \ldots \subset J_{1, S} \subset J_{0, s}=J_{S},
$$

and $s_{i, S}$ is an isomorphism $\Omega_{X}^{i} \otimes \mathcal{O}_{S} \simeq J_{i, S} / J_{i+1, S}, i=0, \ldots, n-1$. Morphisms are isomorphisms of the corresponding $\mathcal{O}_{X \times S}$-modules making all natural diagrams commutative.

We have a natural representable morphism of stacks $\widetilde{\nu}: \widetilde{Q} \rightarrow \mathcal{M}_{n}^{\prime}$, which for each $\mathbb{F}_{q}$-scheme $S$ maps $\left\{L_{S}, \mathbf{s}_{S}, J_{S},\left(J_{i, S}\right),\left(s_{i, S}\right)\right\}$ to the pair $\left\{L_{S}, \mathbf{s}_{S} \circ s_{n-1, S}\right\}$, where $s_{n-1, S}$ is viewed as an embedding of $\Omega_{X}^{n} \otimes \mathcal{O}_{S}$ into $J_{S}$.

Let $\widetilde{Q}=\widetilde{Q}\left(\mathbb{F}_{q}\right)$ be the set of $\mathbb{F}_{q}$-points of $\widetilde{Q}$ (see Sect. 1.7). By definition, it consists of quintuples $\left\{L, \mathbf{s}, J,\left(J_{i}\right),\left(s_{i}\right)\right\}$, where $L$ and $J$ are rank $n$ bundles on $X$, $\mathbf{s}: J \rightarrow L$ is an embedding of the corresponding $\mathcal{O}_{X}$-modules, $\left(J_{i}\right)$ is a full flag of subbundles

$$
0=J_{n} \subset J_{n-1} \subset \ldots \subset J_{1} \subset J_{0}=J
$$

and $s_{i}$ is an isomorphism $s_{i}: \Omega^{i} \simeq J_{i} / J_{i+1}, i=0, \ldots, n-1$.

There is a natural map of sets $r: \widetilde{Q} \rightarrow Q$ defined as follows. Given an object $\left\{L, \mathbf{s}, J,\left(J_{i}\right),\left(s_{i}\right)\right\}$, define $F_{i}$ to be the maximal locally free submodule of $L$ of rank $n-i$, which contains the image of $J_{i} \subset J$ under $\mathbf{s}$. Then $\left(F_{i}\right)$ is a full flag of subbundles of $L$. The composition of $s_{i}: \Omega^{i} \rightarrow J_{i} / J_{i+1}$ with the natural map $J_{i} / J_{i+1} \rightarrow F_{i} / F_{i+1}$ induced by $\mathbf{s}$ is an $\mathcal{O}$-module homomorphism $s_{i}^{\prime}: \Omega^{i} \rightarrow F_{i} / F_{i+1}$ for each $i=0, \ldots, n-1$. Then $\left\{L,\left(F_{i}\right),\left(s_{i}^{\prime}\right)\right\}$ is a point of $Q$. Thus we obtain a $\operatorname{map} r: \widetilde{Q} \rightarrow Q$.

Lemma 3.1. The composition $\nu \circ r: \widetilde{Q} \rightarrow M_{n}^{\prime}$ coincides with the map $\widetilde{\nu}$. Moreover for every function $f$ on $\widetilde{Q}$ we have $\nu_{!}\left(r_{!}(f)\right)=\widetilde{\nu}_{!}(f)$, the integrations being taken with respect to the canonical measures on each of the three sets.

3.3. The sheaf $\mathcal{L}_{E}$. In this section we recall Laumon's construction [22] of the sheaf $\mathcal{L}_{E}$. Let Coh be the stack classifying torsion sheaves of finite length on $X$, i.e., for an $\mathbb{F}_{q}$-scheme $S$, $\operatorname{Hom}(S$, Coh $)$ is the groupoid whose objects are coherent sheaves $\mathcal{T}_{S}$ on $X \times S$, which are finite and flat over $S$ (see $[22]^{2}$ ).

Let $\mathrm{Coh}_{n}$ be the open substack of Coh that classifies torsion sheaves that have at most $n$ indecomposable summands supported at each point. The stack $\operatorname{Coh}_{n}$ can be understood as follows. Let $K$ be a field containing $\mathbb{F}_{q}$, and let $\mathcal{T} \in \operatorname{Coh}(K)$. We have a (non-canonical) isomorphism

$$
\mathcal{T} \simeq \mathcal{O}_{X_{K}} / \mathcal{O}_{X_{K}}\left(-D_{1}\right) \oplus \cdots \oplus \mathcal{O}_{X_{K}} / \mathcal{O}_{X_{K}}\left(-D_{h}\right)
$$

where $X_{K}=\operatorname{Spec} K \times_{\operatorname{Spec} \mathbb{F}_{q}} X$, and $D_{1} \geq D_{2} \geq \cdots \geq D_{h}$ is a decreasing sequence (uniquely determined by $\mathcal{T}$ ) of effective divisors on $X_{K}$. The torsion sheaf $\mathcal{T}$ belongs to $\operatorname{Coh}_{n}(K)$ precisely when $h \leq n$. Let $S$ be an $\mathbb{F}_{q}$-scheme. Then a torsion sheaf $\mathcal{T}_{S} \in \operatorname{Coh}(S)$ belongs to $\operatorname{Coh}_{n}(S)$ if it does so at every closed point of $S$.

\footnotetext{
${ }^{2}$ The notation used in [22] is $\operatorname{Coh}^{0}$; we suppress the upper index 0 to simplify notation.
} 
The stack $\mathrm{Coh}_{n}$ is a disjoint union of connected components

$$
\operatorname{Coh}_{n}=\bigcup_{m \in \mathbb{Z}_{+}} \operatorname{Coh}_{n, m}
$$

where the component $\operatorname{Coh}_{n, m}$ classifies torsion sheaves of degree $m$; the degree of the torsion sheaf $\mathcal{T}$ in (3.1) is $\sum_{i} \operatorname{deg}\left(D_{i}\right)$. Each $\operatorname{Coh}_{n, m}$ has an open substack $\operatorname{Coh}_{n, m}^{r s s}$ classifying regular semi-simple torsion sheaves: an $S$-point $\mathcal{T}_{S}$ of $\operatorname{Coh}_{n}$ is said to be a point of $\operatorname{Coh}_{n, m}^{r s s}$ if for any $\overline{\mathbb{F}}_{q}$-point of $S$ the corresponding sheaf over $X \underset{\operatorname{Spec} \mathbb{F}_{q}}{\times} \operatorname{Spec} \overline{\mathbb{F}}_{q}$ is isomorphic to $\bigoplus \mathcal{O}_{X} / \mathcal{O}_{X}\left(-x_{i}\right)$, where the points $x_{i}$ are distinct.

Let $X^{(m)}$ denote the $m$ th symmetric power of $X$ and let $X^{(m), r s s}$ be the complement to the divisor of diagonals in $X^{(m)}$. We have a smooth map $X^{(m), r s s} \rightarrow$ Coh $h_{n, m}^{r s s}$. When we make base change from $\mathbb{F}_{q}$ to $\overline{\mathbb{F}}_{q}$, Coh $h_{n, m}^{r s s}$ can be identified with the quotient of $X^{(m)}$ by $G L_{1}^{m}$ with respect to the trivial action.

Let us consider the rank $n$ local system $E=E_{\sigma}$ on $X$ corresponding to the Galois representation $\sigma$. Let us also write $\pi: X^{m} \rightarrow X^{(m)}$ for the natural projection. Define the $m$ th symmetric power $E^{(m)}$ of $E$ as the sheaf of invariants of $\pi_{*} E^{\bigotimes m}$ under the natural action of the symmetric group $S_{m}$, i.e., $E^{(m)}=\left(\pi_{*} E^{\bigotimes m}\right)^{S_{m}}$. The restriction $\left.E^{(m)}\right|_{X^{(m), r s s}}$ clearly descends to a local system $\mathcal{L}_{E, m}^{0}$ on $\operatorname{Coh}_{n, m}^{r s s}$, since it does over the algebraic closure of $\mathbb{F}_{q}$. We define the perverse sheaf $\mathcal{L}_{E}$ as the sheaf on $\operatorname{Coh}_{n}$, whose restriction to each $\operatorname{Coh}_{n, m}$ is the Goresky-MacPherson extension of $\mathcal{L}_{E, m}^{0}$, i.e., $\mathcal{L}_{E} \mid \operatorname{eoh}_{n, m}=j_{!_{*}} \mathcal{L}_{E, m}^{0}$, where $j: \operatorname{Coh}_{n, m}^{r s s} \hookrightarrow \operatorname{Coh}_{n, m}$.

We will need an explicit description of the function $L_{E}$ corresponding to $\mathcal{L}_{E}$ on the set $\operatorname{Coh}_{n, m}$. Let $x \in|X|$ be a closed point with residue field $k_{x}$. Then we can regard $x$ as a $k_{x}$-rational point of $X$. Recall from Sect. 2.1 that we denote by $q_{x}$ the cardinality of $k_{x}$, and that $q_{x}=q^{\operatorname{deg} x}$. We denote by $\operatorname{Coh}_{n, m}(x)$ the algebraic stack over $k_{x}$ that classifies torsion sheaves of degree $m$ on $X \underset{\text { Spec } \mathbb{F}_{q}}{\times}$ Spec $k_{x}$ supported at $x$ that have at most $n$ indecomposable summands. Obviously, $\operatorname{Coh}_{n, m}(x)$ is a locally closed substack of $\mathcal{C o h} h_{n, m} \underset{\operatorname{Spec} \mathbb{F}_{q}}{\times}$ Spec $k_{x}$; we denote by $I_{m, x}$ the corresponding embedding. Let $\mathcal{L}_{E, m, x}$ be the pull-back to $\operatorname{Coh}_{n, m}(x)$ of the sheaf $\mathcal{L}_{E, m}$ under the composition

$$
\operatorname{Coh}_{n, m}(x) \stackrel{I_{m, x}}{\longrightarrow} \operatorname{Coh}_{n, m} \underset{\operatorname{Spec} \mathbb{F}_{q}}{\times} \operatorname{Spec} k_{x} \rightarrow \operatorname{Coh}_{n, m} .
$$

We will denote the corresponding function by $L_{E, m, x}$.

In what follows, we fix, once and for all, a geometric point $\bar{x}$ over each closed point $x \in|X|$. We denote by $E_{x}$ the stalk of $E$ at $\bar{x}$.

Let $P_{n, m}^{++}$be the set

$$
\left\{\lambda=\left(\lambda_{1}, \lambda_{2}, \ldots, \lambda_{n}\right) \mid \lambda_{i} \in \mathbb{Z}, \lambda_{1} \geq \lambda_{2} \geq \ldots \geq \lambda_{n} \geq 0, \sum_{i} \lambda_{i}=m\right\} .
$$

We can consider $P_{n, m}^{++}$as a subset of the set $P_{n}^{+}$of dominant weights of $G L_{n}$. For $\lambda \in P_{n, m}^{++}$, we write $E_{x}(\lambda)$ for the representation of $G L_{n}\left(\overline{\mathbb{Q}}_{\ell}\right) \cong G L\left(E_{x}\right)$ of highest weight $\lambda$.

The stack $\operatorname{Coh}_{n, m}(x)$ has a stratification by locally closed substacks $\operatorname{Coh}_{n, m}^{\lambda}(x)$ indexed by $\lambda \in P_{n, m}^{++}$. The stratum corresponding to $\lambda=\left(\lambda_{1}, \lambda_{2}, \ldots, \lambda_{n}\right) \in P_{n, m}^{++}$ parametrizes torsion sheaves of the form

$$
\mathcal{T} \simeq \mathcal{O}_{X} / \mathcal{O}_{X}\left(-\lambda_{1} x\right) \oplus \cdots \oplus \mathcal{O}_{X} / \mathcal{O}_{X}\left(-\lambda_{n} x\right) .
$$


Let $\mathcal{B}_{\lambda, x}$ denote the intersection cohomology sheaf associated to the constant sheaf on the stratum $\operatorname{Coh}_{n, m}^{\lambda}(x)$.

Let $\mathcal{T}$ be an $\mathbb{F}_{q}$-valued point of $\mathcal{C} o h_{n, m}$ and let $x \in|X|$ be a closed point with residue field $k_{x}$. The pull-back of $\mathcal{T}$ to a sufficiently small Zariski neighborhood of $x$ in $X \underset{\operatorname{Spec} \mathbb{F}_{q}}{\times} \operatorname{Spec} k_{x}$ gives rise to an object $\mathcal{T}_{x}$ of $\mathcal{C} o h_{n, m_{x}}(x)$, i.e., to a $k_{x}$-rational point of $\operatorname{Coh}_{n, m_{x}}(x)$, for some $m_{x}$. We have: $\sum_{x \in|X|} m_{x} \cdot \operatorname{deg}(x)=m$. Moreover, there is a bijection:

$$
\operatorname{Coh}_{n}\left(\mathbb{F}_{q}\right)=\prod_{x \in|X|}^{\prime} \operatorname{Coh}_{n}(x)\left(k_{x}\right) .
$$

The explicit description of $L_{E}$ is given in the following proposition. Note that the shifts in degrees are due to the fact that the stack $\operatorname{Coh}_{n, m}(x)$ has dimension $-m$, whereas the dimension of $\operatorname{Coh}_{n, m}$ is zero.

Proposition 3.1 ([22], (3.3.8)). (1) Let $\mathcal{T}$ be an $\mathbb{F}_{q}$-point of $\mathcal{C o h}_{n, m}$. Then, using the notation above, we have:

$$
L_{E, m}(\mathcal{T})=\prod_{x \in|X|} L_{E, m_{x}, x}\left(\mathcal{T}_{x}\right) .
$$

(2) Furthermore,

$$
\mathcal{L}_{E, m, x} \simeq \bigoplus_{\lambda \in P_{n, m}^{++}} \mathcal{B}_{\lambda, x}[m](-n(\lambda)) \otimes E_{x}(\lambda),
$$

where $n(\lambda)=\sum_{i=1}^{n}(i-1) \lambda_{i}$.

Remark 3.1. Let $x$ be an $\mathbb{F}_{q}$-rational point of $X$. Consider now the variety $\mathcal{N}_{m} \subset$ $\mathfrak{g l}_{m}$ of nilpotent matrices. The stack $\operatorname{Coh}_{m, m}(x)$ is isomorphic to the stack $\mathcal{N}_{m} / G L_{m}$, where $G L_{m}$ acts on $\mathcal{N}_{m}$ by conjugation. Let $\pi: \widetilde{\mathcal{N}}_{m} \rightarrow \mathcal{N}_{m}$ denote the Springer resolution and let $\mathcal{S} p_{m}=R \pi_{*} \overline{\mathbb{Q}}_{\ell}$ denote the Springer sheaf on $\mathcal{N}_{m}$. The sheaf $\delta p_{m}$ has a natural action of the symmetric group $S_{m}$. It is shown in [22] that

$$
\left.\mathcal{L}_{E, m, x} \simeq\left(\mathcal{S} p_{m} \otimes\left(E_{x}\right)^{\otimes m}\right)^{S_{m}}\right|_{e h_{n, m}}
$$

(note that $\operatorname{Coh}_{n, m}$ is an open substack of $\left(o h_{m, m}\right.$ ). Hence the function $L_{E}$ associated to $\mathcal{L}_{E}$ can be expressed via the Kostka-Foulkes polynomials; see [22].

However, it will be more convenient for us to use another interpretation of the sheaf $\mathcal{L}_{E, m, x}$, via the affine Grassmannian (see Sect. 4.2). This interpretation allows us to express $L_{E}$ in terms of the Hecke algebra $\mathcal{H}\left(G L_{n}(\mathcal{K}), G L_{n}(\mathcal{O})\right)$; see Sect. 5.5. The fact that the two interpretations agree is due to Lusztig [26].

3.4. The sheaf $\mathcal{F}_{E}$. Define a morphism of stacks $\alpha: \widetilde{\mathcal{Q}} \rightarrow \operatorname{Coh}_{n}$ that sends a quintuple $\left\{L_{S}, \mathbf{s}_{S}, J_{S},\left(J_{i, S}\right),\left(s_{i, S}\right)\right\}$ to the sheaf $L_{S} / \operatorname{Im} \mathbf{s}_{S}$.

Now we define a morphism $\beta: \widetilde{\mathcal{Q}} \rightarrow \mathbb{G}_{a, \mathbb{F}_{q}}$, which at the level of $\mathbb{F}_{q}$-points sends $\left\{L, \mathbf{s}, J,\left(J_{i}\right),\left(s_{i}\right)\right\}$ to the sum of $n-1$ classes in

$$
\mathbb{F}_{q} \simeq \operatorname{Ext}^{1}\left(\Omega^{i}, \Omega^{i-1}\right) \simeq \operatorname{Ext}^{1}\left(J_{i} / J_{i+1}, J_{i-1} / J_{i}\right)
$$

that correspond to the successive extensions

$$
0 \rightarrow J_{i-1} / J_{i} \rightarrow J_{i-1} / J_{i+1} \rightarrow J_{i} / J_{i+1} \rightarrow 0 .
$$


Given two coherent sheaves $L$ and $L^{\prime}$ on $X$, consider the stack $\mathcal{E} x t^{1}\left(L^{\prime}, L\right)$, such that the objects of the groupoid $\operatorname{Hom}\left(S, \mathcal{E} x t^{1}\left(L^{\prime}, L\right)\right)$ are coherent sheaves $L^{\prime \prime}$ on $X \times S$ together with a short exact sequence

$$
0 \rightarrow L \otimes \mathcal{O}_{S} \rightarrow L^{\prime \prime} \rightarrow L^{\prime} \otimes \mathcal{O}_{S} \rightarrow 0
$$

and morphisms are morphisms between such exact sequences inducing isomorphisms at the ends. There is a canonical morphism of stacks $\mathcal{E} x t^{1}\left(L^{\prime}, L\right) \rightarrow$ $\operatorname{Ext}^{1}\left(L^{\prime}, L\right)$. We have for each $i=1, \ldots, n-1$, a natural morphism $\beta_{i}: \widetilde{\mathcal{Q}} \rightarrow$ $\mathcal{E} x t^{1}\left(\Omega^{i}, \Omega^{i-1}\right)$, as above. Now $\beta$ is the composition

$$
\widetilde{\mathcal{Q}} \rightarrow \prod_{i=1}^{n-1} \varepsilon x t^{1}\left(\Omega^{i}, \Omega^{i-1}\right) \rightarrow \prod_{i=1}^{n-1} E x t^{1}\left(\Omega^{i}, \Omega^{i-1}\right) \rightarrow \mathbb{G}_{a, \mathbb{F}_{q}}^{n-1} \rightarrow \mathbb{G}_{a, \mathbb{F}_{q}}
$$

Let $\mathcal{J}_{\psi}$ be the Artin-Schreier sheaf on $\mathbb{G}_{a, \mathbb{F}_{q}}$ corresponding to the character $\psi$.

Recall that the Galois representation $\sigma$ gives rise to a rank $n$ local system $E$ on $X$ and to the sheaf $\mathcal{L}_{E}$ on $\operatorname{Coh}_{n}$. Define the sheaf $\mathcal{F}_{E}$ on $\widetilde{\mathcal{Q}}$ as

$$
\mathcal{F}_{E}:=\alpha^{*}\left(\mathcal{L}_{E}\right) \otimes \beta^{*}\left(\mathcal{J}_{\psi}\right)
$$

Note that $\widetilde{\mathcal{Q}}$ is an open substack in a vector bundle over the product of Coh $_{n}$ and a smooth stack that classifies extensions $J$ as above. Hence the map $\alpha$ is smooth, and $\mathcal{F}_{E}$ is the Goresky-MacPherson extension from its restriction to the open substack $\alpha^{-1}\left(\operatorname{Coh}_{n}^{r s s}\right)$.

3.5. Geometric Langlands conjecture for $G L_{n}$. Recall that we have a representable morphism of stacks $\widetilde{\nu}: \widetilde{Q} \rightarrow \mathcal{M}_{n}^{\prime}$ that associates the pair $\left\{L, \mathbf{s} \circ s_{n-1}\right\}$ to an object $\left\{L, \mathbf{s}, J,\left(J_{i}\right),\left(s_{i}\right)\right\}$.

We define the complex of $\ell$-adic sheaves $\mathcal{S}_{E}^{\prime}$ on $\mathcal{M}_{n}^{\prime}$ to be the direct image

$$
\mathcal{S}_{E}^{\prime}:=\widetilde{\nu}_{!}\left(\mathcal{F}_{E}\right) \text {. }
$$

The following conjecture of Laumon is a geometric version of Conjecture 2.3.

Conjecture $3.1([25])$. Let $\sigma$ be in $\mathfrak{G}_{n}$ and $E$ be the corresponding irreducible $\ell-$ adic local system on $X$. Then

- The restriction of the complex $\mathcal{S}_{E}^{\prime}$ to each connected component of $\mathcal{M}_{n}^{\prime}$ is an irreducible perverse sheaf up to a shift in degree.

- $\mathcal{S}_{E}^{\prime} \simeq p^{*}\left(\mathcal{S}_{E}\right)$, where $p$ is the natural morphism $\mathcal{M}_{n}^{\prime} \rightarrow \mathcal{M}_{n}$, and $\mathcal{S}_{E}$ is a complex of sheaves on $\mathcal{M}_{n}$, whose restriction to each connected component of $\mathcal{M}_{n}$ is an irreducible perverse sheaf up to a shift.

- The sheaf $\mathcal{S}_{E}$ is an eigensheaf of the Hecke correspondences in the sense of [22], (2.1.1).

If this conjecture is true, then the function on $M_{n}$ associated to the sheaf $\mathcal{S}_{E}$ is the automorphic function $f_{\sigma}$ corresponding to $\sigma$. The sheaf $\mathcal{S}_{E}$ can therefore be called the automorphic sheaf corresponding to $\sigma$.

The conjecture means that the sheaf $\mathcal{S}_{E}^{\prime}$ is constant along the fibers of the morphism $p$. Thus, it is analogous to Conjecture 2.3. The advantage of dealing with Conjecture 3.1 as compared to Conjecture 2.3 is that while the latter is a global statement, one could use local geometric information about the sheaf $\mathcal{S}_{E}^{\prime}$ to tackle Conjecture 3.1 (as Drinfeld did in the case of $G L_{2}[7]$ ). 
Remark 3.2. The above conjecture is obviously false if one does not assume the irreducibility of $\sigma$ (the complex $\mathcal{S}_{E}^{\prime}$ must be corrected by the corresponding "constant terms" in this case). However one can construct the automorphic sheaves $\mathcal{S}_{E}$ corresponding to $\sigma$ 's, which are direct sums of one-dimensional representations, by means of the geometric Eisenstein series [23].

3.6. Main theorem. Let $S_{E}^{\prime}$ denote the function on $M_{n}^{\prime}$ associated to $\mathcal{S}_{E}^{\prime}$. If Conjecture 3.1 is true, then this function has the same properties as the function $f_{\sigma}^{\prime}$ defined in Sect. 2.3. Recall that these properties uniquely determine $f_{\sigma}^{\prime}$ up to a non-zero factor. Therefore Conjecture 3.1 can be true only if the functions $S_{E}^{\prime}$ and $f_{\sigma}^{\prime}$ are proportional. This was conjectured by Laumon in [25] (Conjecture 3.2). One of our motivations was to prove this conjecture. More precisely, we prove the following:

Theorem 3.1. The functions $S_{E}^{\prime}$ and $f_{\sigma}^{\prime}$ are equal.

Theorem 3.1 means that the function $f_{\sigma}^{\prime}$ does come from a complex of $\ell$-adic sheaves on $\mathcal{M}_{n}^{\prime}$. It also provides a consistency check for Conjecture 3.1. Laumon has proved Theorem 3.1 in [22] for $G L_{2}$ by a method different from the one we use below. We derive Theorem 3.1 for $G L_{n}$ with arbitrary $n$ from the following statement.

Proposition 3.2. Let $F_{E}$ be the function on $\widetilde{Q}$ corresponding to $\mathcal{F}_{E}$. The function $r_{!}\left(F_{E}\right)$ coincides with the restriction of the Whittaker function $W_{\sigma}$ to $Q$.

Theorem 3.1 immediately follows from Proposition 3.2 because of Lemma 3.1 as shown in the diagram below.

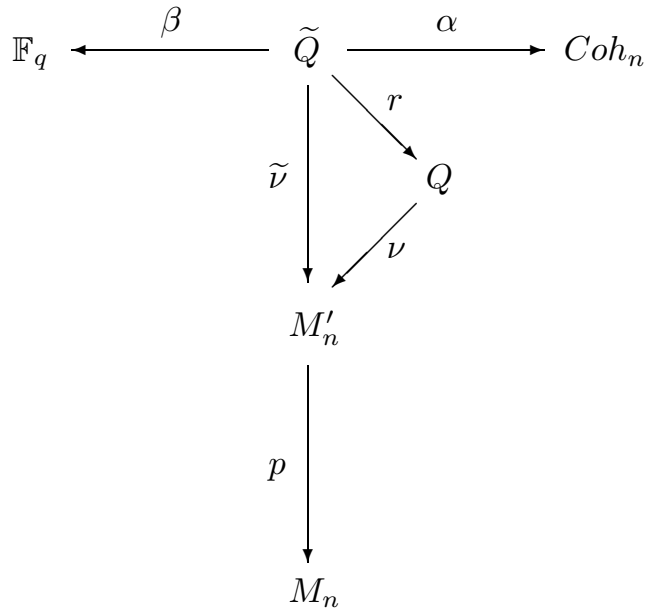

The proof of Proposition 3.2 will occupy Sects. 4 and 5 below.

Proposition 3.2 can be interpreted in the following way. Denote by $\mathrm{Coh}_{n}$ (resp., $\left.C o h_{n, m}(x)\right)$ the set of $\mathbb{F}_{q}$-points (resp., $k_{x}$-points) of Coh $_{n}$ (resp., Coh $h_{n, m}(x)$ ). Set $\operatorname{Coh}_{n}(x)=\bigcup_{m \geq 0} \operatorname{Coh}_{n, m}(x)$. Then by formula (3.2), $\operatorname{Coh}_{n}=\prod_{x \in|X|}^{\prime} \operatorname{Coh}_{n}(x)$. We have:

$$
\operatorname{Coh}_{n}(x) \simeq P_{n}^{++}=\bigcup_{m \geq 0} P_{n, m}^{++} .
$$

Hence we can identify $\operatorname{Coh}_{n}(x)$ with the set

$$
\left\{\operatorname{diag}\left(\pi_{x}^{\lambda_{1}}, \ldots, \pi_{x}^{\lambda_{n}}\right) \mid \lambda_{1} \geq \ldots \geq \lambda_{n} \geq 0\right\} .
$$


The Whittaker function $W_{\sigma}$ can then be restricted to the $C o h_{n}$, and it is uniquely determined by this restriction; see Sect. 2.2. Thus, both $L_{E}$ and $W_{\sigma}$ give rise to functions on $C o h_{n}$. For each point $t \in C o h_{n}$, the value of $L_{E}$ at $t$ is given by taking the alternating sum of traces of the Frobenius on the stalk cohomologies of $\mathcal{L}_{E}$ at $t$. On the other hand, the value of $W_{\sigma}$ is given by the trace of the Frobenius on the top stalk cohomology of $\mathcal{L}_{E}$ at $t$ (see [22]). Therefore Proposition 3.2 says that the contributions of all stalk cohomologies, other than the top one, are killed by the summation along the fibers of the projection $r$ against the non-trivial character $\Psi$.

Remark 3.3. As we mentioned in the introduction, Drinfeld has proved a version of Conjecture 3.1 for $G L_{2}$. The case of $G L_{2}$ is special as explained below.

Let $\widetilde{\mathcal{M}}_{2}^{\prime}$ be the open substack of $\mathcal{M}_{2}^{\prime}$, which parametrizes $\{L, s\}$, such that the image of $s: \Omega \rightarrow L$ is a maximal invertible subsheaf of $L$. Due to the Hecke eigenfunction property of $f_{\sigma}^{\prime}$ with respect to $T_{x}^{2}, f_{\sigma}^{\prime}$ is uniquely determined by its restriction to $\widetilde{M}_{2}^{\prime}=\widetilde{\mathcal{M}}_{2}^{\prime}\left(\mathbb{F}_{q}\right)$.

But the map $r$ is a bijection over $\nu^{-1}\left(\widetilde{M}_{2}^{\prime}\right)$, and hence $\nu^{-1}\left(\widetilde{M}_{2}^{\prime}\right)$ can be considered as a subset of $\widetilde{Q}$. Clearly, the map $\alpha$ of Sect. 3.3 sends $\nu^{-1}\left(\widetilde{M}_{2}^{\prime}\right)$ to $\mathrm{Coh}_{1} \subset \mathrm{Coh}_{2}$. Furthermore, the restriction of $\mathcal{L}_{E}$ to the stack $\operatorname{Coh}_{1}$ is simply a sheaf, i.e., it has stalk cohomology in only one degree. Therefore on $C o h_{1}$ the function $L_{E}$ equals the Whittaker function $W_{\sigma}$. Hence, restricted to $\widetilde{\mathcal{M}}_{2}^{\prime}$, the geometric construction evidently coincides with the construction described in Sect. 2.3.

\section{Reduction to A LOCAL STATEMENT}

4.1. Adelic interpretation of $\widetilde{Q}$. Recall that $\widetilde{Q}$ is the set of isomorphism classes of $\mathbb{F}_{q}$-points of $\widetilde{Q}$, and in Sect. 3.1 we defined a map $r: \widetilde{Q} \rightarrow Q$. Recall further that $\operatorname{Coh}_{n}(x) \simeq P_{n}^{++}=\bigcup_{m \geq 0} P_{n, m}^{++}$, and $\operatorname{Coh}_{n}=\prod_{x \in|X|}^{\prime} \operatorname{Coh}_{n}(x)$.

Denote $G L_{n}^{J}\left(\mathcal{K}_{x}\right)^{+}=G L_{n}^{J}\left(\mathcal{K}_{x}\right) \cap \operatorname{Mat}_{n}^{J}\left(\mathcal{O}_{x}\right)$. Let $G L_{n}^{J}(\mathbb{A})^{+}$be the restricted product $\prod_{x \in|X|}^{\prime} G L_{n}^{J}\left(\mathcal{K}_{x}\right)^{+}$. We have bijections:

$$
G L_{n}^{J}\left(\mathcal{O}_{x}\right) \backslash G L_{n}^{J}\left(\mathcal{K}_{x}\right)^{+} / G L_{n}^{J}\left(\mathcal{O}_{x}\right) \simeq \operatorname{Coh}_{n}(x)
$$

and

$$
G L_{n}^{J}(\mathcal{O}) \backslash G L_{n}^{J}(\mathbb{A})^{+} / G L_{n}^{J}(\mathcal{O}) \simeq C o h_{n} .
$$

Proposition 4.1. (1) There is a bijection between the set $\widetilde{Q}$ and the set

$$
\left(N^{J}(F) \backslash N^{J}(\mathbb{A})\right) \underset{N^{J}(\mathcal{O})}{\times}\left(G L_{n}^{J}(\mathbb{A})^{+} / G L_{n}^{J}(\mathcal{O})\right) .
$$

The group $N^{J}(\mathcal{O})$ acts on the product $\left(N^{J}(F) \backslash N^{J}(\mathbb{A})\right) \times\left(G L_{n}^{J}(\mathbb{A})^{+} / G L_{n}^{J}(\mathcal{O})\right)$ according to the rule $y \cdot(u, g)=\left(u \cdot y^{-1}, y \cdot g\right)$.

(2) The map $r: \widetilde{Q} \rightarrow Q$ identifies with the map

$$
\left(N^{J}(F) \backslash N^{J}(\mathbb{A})\right) \underset{N^{J}(\mathcal{O})}{\times}\left(G L_{n}^{J}(\mathbb{A})^{+} / G L_{n}^{J}(\mathcal{O})\right) \rightarrow Q \subset N^{J}(F) \backslash G L_{n}^{J}(\mathbb{A}) / G L_{n}^{J}(\mathcal{O})
$$

given by $(u, g) \rightarrow u \cdot g$.

The map $\alpha$ sends $(u, g)$ to the image of $g$ in $G L_{n}^{J}(\mathcal{O}) \backslash G L_{n}^{J}(\mathbb{A})^{+} / G L_{n}^{J}(\mathcal{O}) \simeq C o h_{n}$.

The map $\beta: \widetilde{Q} \rightarrow \mathbb{F}_{q}$ is the composition of the natural map $N^{J}(F) \backslash N^{J}(\mathbb{A}) / N^{J}(\mathcal{O})$ $\rightarrow \mathbb{F}_{q}^{n-1}$ and the summation $\mathbb{F}_{q}^{n-1} \rightarrow \mathbb{F}_{q}$. 
Proof. We will use the notation introduced in the proof of Lemma 2.1. Let $\{L, \mathbf{s}, J$, $\left.\left(J_{i}\right),\left(s_{i}\right)\right\}$ be an element of $\widetilde{Q}$. Then the triple $\left\{J,\left(J_{i}\right),\left(s_{i}\right)\right\}$ is an element of $Q$. Hence we can associate to it homomorphisms $\varphi_{x}^{J}: J_{\mathcal{K}_{x}}^{0} \rightarrow J_{\mathcal{K}_{x}}, \varphi_{\text {gen }}^{J}: J_{\mathcal{K}_{x}}^{0} \rightarrow J_{\mathcal{K}_{x}}$ and $\mu_{x}^{J}=\left(\varphi_{x}^{J}\right)^{-1} \varphi_{\text {gen }}^{J}: J_{\mathcal{K}_{x}}^{0} \rightarrow J_{\mathcal{K}_{x}}^{0}$, as in the proof of Lemma 2.1.

On the other hand, let $\varphi_{x}^{L}$ be an isomorphism $J_{\mathcal{O}_{x}}^{0} \rightarrow L_{\mathcal{O}_{x}}$. We extend it to a homomorphism $J_{\mathcal{K}_{x}}^{0} \rightarrow L_{\mathcal{K}_{x}}$, which we denote by the same symbol. Denote by $\mathbf{s}_{x}$ the homomorphism $J_{\mathcal{K}_{x}} \rightarrow L_{\mathcal{K}_{x}}$ induced by s. Consider the automorphism $\nu_{x}=\left(\varphi_{x}^{L}\right)^{-1} \mathbf{s}_{x} \varphi_{x}^{J}$ of $J_{\mathcal{K}_{x}}^{0}$.

Now we assign to $\left\{L, \mathbf{s}, J,\left(J_{i}\right),\left(s_{i}\right)\right\}$ the element $\left(\left(u_{x}\right),\left(g_{x}\right)\right)$ of $G L_{n}^{J}(\mathbb{A}) \times G L_{n}^{J}(\mathbb{A})$, where $u_{x}$ is the transpose of the matrix representing $\mu_{x}$, and $g_{x}$ is the transpose of the matrix representing $\nu_{x}$ (see the proof of Lemma 2.1). By construction, $u_{x} \in N^{J}\left(\mathcal{K}_{x}\right)$ and $g_{x} \in G L_{n}^{J}\left(\mathcal{K}_{x}\right)^{+}$. Furthermore, the arbitrariness in the choice of $\varphi_{\text {gen }}^{J}$ corresponds to left multiplication of $u_{x}$ by elements of $N^{J}(F)$, the arbitrariness in $\varphi_{x}^{L}$ corresponds to right multiplication of $g_{x}$ by elements of $G L_{n}^{J}\left(\mathcal{O}_{x}\right)$, and the arbitrariness in $\varphi_{x}^{J}$ corresponds to the action of $N^{J}\left(\mathcal{O}_{x}\right)$ on $\left(u_{x}, g_{x}\right)$ according to the rule $y \cdot\left(u_{x}, g_{x}\right)=\left(u_{x} \cdot y^{-1}, y \cdot g_{x}\right)$.

This proves part (1) of the proposition. The proof of part (2) is now straightforward.

Recall that $L_{E}$ is the function on $C o h_{n}$ associated to the sheaf $\mathcal{L}_{E}$. Denote by $L_{E, x}$ the function on $\operatorname{Coh}_{n}(x)$ whose restriction to $\operatorname{Coh}_{n, m}(x)\left(\mathbb{F}_{q}\right)$ is the function associated to $\mathcal{L}_{E, m, x}$. Part (1) of Proposition 3.1 implies that

$$
L_{E}\left(\left(\lambda_{x}\right)\right)=\prod_{x \in|X|} L_{E, x}\left(\lambda_{x}\right)
$$

for all $\left(\lambda_{x}\right) \in \prod_{x \in|X|}^{\prime} P_{n}^{++}$. Using the bijection (4.1) (resp., (4.2)) we consider $L_{E, x}$ (resp., $L_{E}$ ) as a function on $G L_{n}^{J}\left(\mathcal{K}_{x}\right)^{+}$(resp., $\left.G L_{n}^{J}(\mathbb{A})^{+}\right)$. Let $\Psi_{x}: N^{J}\left(\mathcal{K}_{x}\right) \rightarrow \overline{\mathbb{Q}}_{\ell}^{\times}$ be the character defined in Sect. 2.2. Note that the function $\Psi_{x}$ (resp., $L_{E, x}$ ) is right (resp., left) $N^{J}\left(\mathcal{O}_{x}\right)$-invariant.

Recall further that $F_{E}$ is the function on $\widetilde{Q}$ associated to the sheaf $\mathcal{F}_{E}$. We conclude:

Lemma 4.1. Under the isomorphism of Proposition 4.1,

$$
F_{E}=\prod_{x \in|X|} \Psi_{x} \times L_{E, x} .
$$

Let us extend the function $L_{E, x}$ by zero from $G L_{n}^{J}\left(\mathcal{K}_{x}\right)^{+}$to $G L_{n}^{J}\left(\mathcal{K}_{x}\right)$. Then Proposition 4.1 and Lemma 4.1 imply:

$$
\left(r_{!} F_{E}\right)\left(\left(g_{x}\right)\right)=\prod_{x \in|X|} \sum_{u_{x} \in N^{J}\left(\mathcal{K}_{x}\right) / N^{J}\left(\mathcal{O}_{x}\right)} L_{E, x}\left(u_{x}^{-1} \cdot g_{x}\right) \Psi_{x}\left(u_{x}\right),
$$

for all $g_{x} \in G L_{n}^{J}\left(\mathcal{K}_{x}\right)^{+}$(each sum is actually finite). Let $d u_{x}$ be the Haar measure on $N^{J}\left(\mathcal{K}_{x}\right)$ normalized so that $\int_{N^{J}\left(\mathcal{O}_{x}\right)} d u_{x}=1$. Using left $G L_{n}^{J}\left(\mathcal{O}_{x}\right)$-invariance of $L_{E, x}$, we can rewrite the last formula as

$$
\left(r_{!} F_{E}\right)\left(\left(g_{x}\right)\right)=\prod_{x \in|X|} \int_{N^{J}\left(\mathcal{K}_{x}\right)} L_{E, x}\left(u_{x}^{-1} \cdot g_{x}\right) \Psi_{x}\left(u_{x}\right) d u_{x}
$$


Proposition 3.2 states that $\left(r_{!} F_{E}\right)\left(\left(g_{x}\right)\right)=\left.W_{\sigma}\left(\left(g_{x}\right)\right)\right|_{Q}$. According to formulas (2.4) and (4.3), this is equivalent to the formula

$$
\int_{N^{J}\left(\mathcal{K}_{x}\right)} L_{E, x}\left(u_{x}^{-1} \cdot g_{x}\right) \Psi_{x}\left(u_{x}\right) d u_{x}=W_{\sigma\left(\mathrm{Fr}_{x}\right)}\left(g_{x}\right),
$$

for all $g_{x} \in G L_{n}^{J}\left(\mathcal{K}_{x}\right)^{+}$. Since both the left and the right hand sides of (4.4) are left $\left(N^{J}\left(\mathcal{K}_{x}\right), \Psi_{x}\right)$-equivariant and right $G L_{n}^{J}(\mathcal{O})$-invariant, it suffices to check formula (4.4) when $g_{x}=\operatorname{diag}\left(\pi^{\nu_{1}}, \ldots, \pi^{\nu_{n}}\right)$, where $\nu=\left(\nu_{1}, \ldots, \nu_{n}\right) \in P_{n}^{++}$.

Using the explicit formula (2.3), we reduce Proposition 3.2 to the following local statement.

\section{Proposition 4.2.}

$$
\int_{N^{J}\left(\mathcal{K}_{x}\right)} L_{E, x}\left(u_{x} \cdot \operatorname{diag}\left(\pi_{x}^{\nu_{1}}, \ldots, \pi_{x}^{\nu_{n}}\right)\right) \Psi^{-1}\left(u_{x}\right) d u_{x}=q_{x}^{n(\nu)} \operatorname{Tr}\left(\sigma\left(\operatorname{Fr}_{x}\right), E_{x}(\nu)\right)
$$

where $\nu \in P_{n}^{++}$.

4.2. Positive part of the affine Grassmannian. Recall that $J^{0}=\bigoplus_{i=0}^{n-1} \Omega^{i}$. From now on we work in the local setting. Hence we choose once and for all a trivialization of $J^{0}$ on the formal neighborhood of $x \in|X|$ and identify $G L_{n}^{J}\left(\mathcal{K}_{x}\right)$ with $G L_{n}\left(\mathcal{K}_{x}\right)$. For this reason we suppress the index $J$ in what follows.

According to part (2) of Proposition 3.1,

$$
L_{E, x}=\sum_{\lambda \in P_{n}^{++}} \operatorname{Tr}\left(\sigma\left(\operatorname{Fr}_{x}\right), E_{x}(\lambda)\right) \cdot B_{\lambda}
$$

where $B_{\lambda}$ is the function on $\operatorname{Coh}_{n}(x)$ associated to the sheaf $\mathcal{B}_{\lambda, x}[m](-n(\lambda)$ ) (see Sect. 3.2). We view $B_{\lambda}$ as a $G L_{n}\left(\mathcal{O}_{x}\right)$-invariant function on $G L_{n}\left(\mathcal{K}_{x}\right) / G L_{n}\left(\mathcal{O}_{x}\right)$.

Let $x$ be a closed point of $X$. To simplify notation, from now on we will assume that $x$ is an $\mathbb{F}_{q}$-rational point of $X$ (otherwise, we simply make a base change from $\mathbb{F}_{q}$ to the residue field $k_{x}$ of $x$ ).

Define the functor which sends an $\mathbb{F}_{q}$-scheme $S$ to the set of isomorphism classes of pairs $\{L, t\}$, where $L$ is a rank $n$ bundle on $X \times S$ and $t$ is its trivialization on $(X \times S)-(\{x\} \times S)$. This functor is representable by an ind-scheme $\mathcal{G} r_{x}$ (see [1]), which we call the affine Grassmannian $\mathcal{G} r_{x}$ (for the group $G L_{n}$ ). The ind-scheme $\mathcal{G} r_{x}$ splits into a disjoint union of connected components: $\mathcal{G} r_{x}=\bigcup_{m \in \mathbb{Z}} \mathcal{G} r_{x}^{m}$ indexed by the degree of $L$ for $\{L, t\} \in \mathcal{G} r_{x}$.

There is a bijection between the set $G r_{x}$ of $\mathbb{F}_{q}$-points of $\mathcal{G} r_{x}$ and the quotient $G L_{n}\left(\mathcal{K}_{x}\right) / G L_{n}\left(\mathcal{O}_{x}\right)$; see [2]. The analogous quotient over the field of complex numbers is known as the affine, or periodic, Grassmannian. This explains the name that we use.

There exists a proalgebraic group $\mathcal{G}\left(\mathcal{O}_{x}\right)$ whose set of $\mathbb{F}_{q}$-points is $G L_{n}\left(\mathcal{O}_{x}\right)$. The group $\mathcal{G}\left(\mathcal{O}_{x}\right)$ acts on $\mathcal{G} r_{x}$, and its orbits stratify $\mathcal{G} r_{x}$ by locally closed finitedimensional subschemes $\mathcal{G} r_{x}^{\lambda}$ indexed by the set $P_{n}^{+}$of dominant weights $\lambda$ of $G L_{n}$. The stratum $\mathcal{G} r_{x}^{\lambda}$ is the $\mathcal{G}\left(\mathcal{O}_{x}\right)$-orbit of the coset $\operatorname{diag}\left(\pi_{x}^{\lambda_{1}}, \ldots, \pi_{x}^{\lambda_{n}}\right) \cdot G L_{n}\left(\mathcal{O}_{x}\right) \in$ $G r_{x}$.

Recall that there is an inner product on the set of $G L_{n}$ weights defined by the formula $(\lambda, \mu)=\sum_{i=1}^{n} \lambda_{i} \mu_{i}$, and that $\operatorname{dim} \mathcal{G} r_{x}^{\lambda}=2(\lambda, \rho)$, where $\rho$ is the half sum of the positive roots of $G L_{n}, \rho=((n-1) / 2,(n-3) / 2, \ldots,-(n-1) / 2)$. Let $\overline{\mathbb{Q}}_{\ell, \lambda}$ be the constant sheaf supported on the stratum $\mathcal{G} r_{x}^{\lambda}$. Denote by $\mathcal{A}_{\lambda, x}$ the intersection 
cohomology sheaf on the closure of $\mathcal{G} r_{x}^{\lambda}$, which is the Goresky-MacPherson extension of the sheaf $\overline{\mathbb{Q}}_{\ell, \lambda}[2(\lambda, \rho)]((\lambda, \rho))$. Let $A_{\lambda}$ be the $G L_{n}\left(\mathcal{O}_{x}\right)$-invariant function on $G L_{n}\left(\mathcal{K}_{x}\right) / G L_{n}\left(\mathcal{O}_{x}\right)$, which is the extension by zero of the function associated to $\mathcal{A}_{\lambda, x}$.

Define now the closed subscheme $\mathcal{G} r_{x}^{+}$of $\mathcal{G} r_{x}$ which at the level of points corresponds to pairs $\{L, t\}$, for which $t$ extends to an embedding of $\mathcal{O}_{X}$-modules $\mathcal{O}_{X}^{\oplus n} \rightarrow L$. The scheme $\mathcal{G} r_{x}^{+}$also splits into a disjoint union of connected components $\mathcal{G} r_{x}^{+}=\bigcup_{m \in \mathbb{Z}^{+}} \mathcal{G} r_{x}^{m,+}$, where $\mathcal{G} r_{x}^{m,+}=\mathcal{G} r_{x}^{+} \cap \mathcal{G} r_{x}^{m}$. It is clear that $\mathcal{G} r_{x}^{m,+}$ is a union of the strata $\mathcal{G r} r_{x}^{\lambda}$, for $\lambda \in P_{n, m}^{++}$(see Sect. 3.2). The set $\mathcal{G r} r_{x}^{+}\left(\mathbb{F}_{q}\right)$ identifies with the quotient $G L_{n}\left(\mathcal{K}_{x}\right)^{+} / G L_{n}\left(\mathcal{O}_{x}\right)$.

Consider the morphism $q_{m, x}: \mathcal{G} r_{x}^{m,+} \rightarrow \operatorname{Coh}_{m, n}(x)$, which sends a pair $\{L, t\}$ to the quotient $\mathcal{O}_{X}^{\oplus n} / \operatorname{Im} t^{*}$, where $t^{*}: L^{*} \rightarrow \mathcal{O}_{X}^{\oplus n}$. This morphism can be described as follows. There is a natural vector bundle $\widetilde{\operatorname{Coh}}_{m, n}$ over the stack $\operatorname{Coh}_{m, n}$, whose fiber at $\mathcal{T}$ is $\operatorname{Hom}\left(\mathcal{O}_{X}^{\oplus n}, \mathcal{T}\right) ; \mathcal{G} r_{x}^{m,+}$ is an open substack of the total space of the bundle $\widetilde{\operatorname{Coh}}_{n}$, which corresponds to epimorphic elements of $\operatorname{Hom}\left(\mathcal{O}_{X}^{\oplus n}, \mathcal{T}\right)$. The map $q_{m, x}$ is simply the projection from this substack to the base. Hence $q_{m, x}$ is a smooth morphism of algebraic stacks. It is clear that it preserves the stratification (compare with [26]). Note that $\operatorname{dim} \mathcal{G} r_{x}^{m,+}=m(n-1)$ and $\operatorname{dim} \operatorname{Coh}_{n, m}(x)=-m$. Therefore

\section{Lemma 4.2.}

$$
q_{m, x}^{*} \mathcal{B}_{\lambda, x}[m](-n(\lambda))=\mathcal{A}_{\lambda, x}[-m(n-1)](-(\lambda, \rho)-n(\lambda)) .
$$

Recall that $m=|\lambda|=\sum_{i=1}^{n} \lambda_{i}$. The lemma implies that as functions on $G L_{n}\left(\mathcal{K}_{x}\right)^{+} / G L_{n}\left(\mathcal{O}_{x}\right)$,

$$
B_{\lambda}=(-1)^{|\lambda|(n-1)} q_{x}^{(\lambda, \rho)+n(\lambda)} A_{\lambda}
$$

Hence, according to (4.6),

$$
L_{E, x}=\sum_{\lambda \in P_{n}^{++}}(-1)^{|\lambda|(n-1)} q_{x}^{(\lambda, \rho)+n(\lambda)} \operatorname{Tr}\left(\sigma\left(\operatorname{Fr}_{x}\right), E_{x}(\lambda)\right) \cdot A_{\lambda} .
$$

This formula will be used in the next section in the proof of Proposition 4.2.

\section{Proof of the Local Statement}

In this section we will state and prove a general result concerning reductive groups over the local non-archimedian field of positive characteristic. In the case of $G L_{n}$ this result implies Proposition 4.2.

5.1. General set-up. Let $G=G(\mathcal{K})$ be a connected, reductive, split algebraic group over the field $\mathcal{K}=\mathbb{F}_{q}((\pi)), T$ - its split maximal torus contained in a Borel subgroup $B$, and $N$ - the unipotent radical of $B$. We again denote by $\mathcal{O}$ the ring of integers of $\mathcal{K}$, by $\pi$ a generator of its maximal ideal, and by $q$ the cardinality of the residue field $k=\mathbb{F}_{q}$. Let $K$ be the compact subgroup $G(\mathcal{O})$ of $G$. We fix a Haar measure of $G$, such that $K$ has measure 1.

Let $\mathcal{H}(G, K)$ denote the Hecke algebra of $G$ with respect to $K$, i.e., $\mathcal{H}(G, K)$ is the algebra of $\overline{\mathbb{Q}}_{\ell}$-valued compactly supported $K$-bi-invariant functions on $G$ with the convolution product:

$$
\left(f_{1} \cdot f_{2}\right)(g)=\int_{G} f_{1}(x) f_{2}\left(g x^{-1}\right) d x
$$


Let ${ }^{L} G$ be the Langlands dual group of $G$ (without the Weil group of $\mathcal{K}$ ), and ${ }^{L} P$ (resp., ${ }^{L} P^{+}$) be the set of weights (resp., dominant weights) of ${ }^{L} G$. Each $\lambda$ can be viewed as a one-parameter subgroup of $G(\mathcal{K})$, and hence $\lambda(\pi)$ is a well-defined element of $G\left(\mathcal{O}_{x}\right)$. We denote by $c_{\lambda}$ the characteristic function of the double coset $K \lambda(\pi) K \subset G$. The functions $c_{\lambda}$ form a basis of $\mathcal{H}(G, K)$.

Let $\operatorname{Rep}{ }^{L} G\left(\overline{\mathbb{Q}}_{\ell}\right)$ denote the Grothendieck ring of the category of finite-dimensional representations of ${ }^{L} G\left(\overline{\mathbb{Q}}_{\ell}\right)$. We consider it as a $\overline{\mathbb{Q}}_{\ell}$-algebra. If $V$ is a finite-dimensional representation of ${ }^{L} G$, denote by $[V]$ the corresponding element of Rep ${ }^{L} G\left(\overline{\mathbb{Q}}_{\ell}\right)$. In particular, for each $\lambda \in{ }^{L} P^{+}$, let $V(\lambda)$ be the finite-dimensional representation of ${ }^{L} G$ with highest weight $\lambda$.

The following statement, often referred to as the Satake isomorphism, is wellknown; see [31], [19], [28], [11].

Theorem 5.1. There is a unique isomorphism $S: \operatorname{Rep}{ }^{L} G\left(\overline{\mathbb{Q}}_{\ell}\right) \rightarrow \mathcal{H}(G, K)$, which maps $[V(\lambda)]$ to

$$
H_{\lambda}=q^{-(\lambda, \rho)}\left(c_{\lambda}+\sum_{\mu \in{ }^{L} P^{+} ; \mu<\lambda} a_{\lambda \mu} c_{\mu}\right), \quad a_{\lambda \mu} \in \mathbb{Z} .
$$

Remark 5.1. Each semi-simple conjugacy class $\gamma$ of the group ${ }^{L} G\left(\overline{\mathbb{Q}}_{\ell}\right)$ defines a homomorphism $\chi_{\gamma}: \operatorname{Rep}{ }^{L} G\left(\overline{\mathbb{Q}}_{\ell}\right) \rightarrow \overline{\mathbb{Q}}_{\ell}$, which maps $[V]$ to $\operatorname{Tr}(\gamma, V)$. We denote the corresponding homomorphism $\mathcal{H} \rightarrow \overline{\mathbb{Q}}_{\ell}$ by the same symbol $\chi_{\gamma}$. This allows us to identify the spectrum of the commutative algebra $\mathcal{H}$ with the set of semi-simple conjugacy classes of ${ }^{L} G\left(\overline{\mathbb{Q}}_{\ell}\right)$. In particular, we have: $\chi_{\gamma}\left(H_{\lambda}\right)=\operatorname{Tr}(\gamma, V(\lambda))$.

5.2. Hecke algebra and the affine Grassmannian. Let again $X$ be as in Sect. 1.1, and $x$ be its $\mathbb{F}_{q}$-point. We define, in the same way as in Sect. 4.2 for $G=G L_{n}$, the ind-scheme $\operatorname{Gr}(G)=\mathcal{G} r(G)_{x}$ that classifies pairs $\{\mathcal{P}, t\}$, where $\mathcal{P}$ is a principal $G$-bundle on $X$ and $t$ is its trivialization over $X-x$. The ind-scheme structure on $\operatorname{Gr}(G)$ is described, e.g., in [20]. Note that due to the results of [2], [8], the global curve $X$ is inessential in the above definition. We could simply take $X=$ Spec $\mathcal{O}$. In particular, there is a bijection between the set of $\mathbb{F}_{q}$-points of $\operatorname{Gr}(G)$ and the set $G / K$.

There is a proalgebraic group $\mathcal{G}(\mathcal{O})$, whose set of $\mathbb{F}_{q}$-points is $G(\mathcal{O})$. This group acts on $\mathcal{G r}(G)$, and its orbits stratify $\mathcal{G} r(G)$ by locally closed finite-dimensional subschemes $\operatorname{Gr}(G)^{\lambda}$ indexed by the set ${ }^{L} P^{+}$of dominant weights $\lambda$ of ${ }^{L} G$. The stratum $\operatorname{Gr}(G)^{\lambda}$ is the $G(\mathcal{O})$-orbit of the coset $\lambda(\pi) \cdot G(\mathcal{O})$, where $\lambda(\pi) \in T(\mathcal{K}) \subset$ $G(\mathcal{K})$ is defined above.

Denote by $(\lambda, \rho)$ the pairing between $\lambda \in{ }^{L} P$ and the sum of the fundamental coweights $\rho$ of ${ }^{L} G$. Let $\overline{\mathbb{Q}}_{\ell, \lambda}$ be the constant sheaf supported on the stratum $\operatorname{Gr}(G)^{\lambda}$. Denote by $\mathcal{A}_{\lambda}=\mathcal{A}_{\lambda, x}$ the intersection cohomology sheaf on the closure of $\operatorname{Gr}(G)^{\lambda}$, which is the Goresky-MacPherson extension of the sheaf $\overline{\mathbb{Q}}_{\ell, \lambda}[2(\lambda, \rho)]((\lambda, \rho)$ ) (note that $\left.\operatorname{dim} \operatorname{Gr}(G)^{\lambda}=2(\lambda, \rho)\right)$. Let $A_{\lambda}$ be the function associated to $\mathcal{A}^{\lambda}$. We use the same notation for its extension by zero to the whole $\operatorname{Gr}(G)$. Clearly, the functions $A_{\lambda}, \lambda \in{ }^{L} P^{+}$, form a basis in the $\overline{\mathbb{Q}}_{\ell}$-vector space $\overline{\mathbb{Q}}_{\ell}(G / K)^{K}$ of $K$-invariant functions on $G / K$ with compact support. We have an isomorphism of vector spaces: $\mathcal{H} \simeq \overline{\mathbb{Q}}_{\ell}(G / K)^{K}$, which commutes with the action of $\mathcal{H}$. Therefore $H_{\lambda} \in \mathcal{H}, \lambda \in$ ${ }^{L} P$, can also be considered as elements of $\overline{\mathbb{Q}}_{\ell}(G / K)^{K}$.

Proposition 5.1. $H_{\lambda}=(-1)^{2(\lambda, \rho)} A_{\lambda}$. 
This result is due to Lusztig [26], [27] and Kato [17] (see, e.g., Theorem 1.8, Lemma 2.7, formula (3.5) of [17]). It implies that

$$
H_{\lambda}(y)=\left.(-1)^{2(\lambda, \rho)} \sum_{i \in \mathbb{Z}} \operatorname{dim} \mathrm{H}^{i}\left(\mathcal{A}_{\lambda}\right)\right|_{y} q^{i / 2},
$$

where $\left.\mathrm{H}^{j}\left(\mathcal{A}_{\lambda}\right)\right|_{y}$ is the $j$ th stalk cohomology of $\mathcal{A}_{\lambda}$ at $y \in G r(G)$.

5.3. Fourier transform. Let us denote by Res : $\mathcal{K} \rightarrow \mathbb{F}_{q}$ the map defined by the formula

$$
\operatorname{Res}\left(\sum_{n \in \mathbb{Z}} f_{i} \pi^{i}\right)=f_{-1} .
$$

We define a character $\Psi$ of $N$ in the following way:

$$
\Psi(u)=\sum_{i=1}^{l} \psi\left(\operatorname{Res}\left(u_{i}\right)\right),
$$

where $u_{i}, i=1, \ldots, l=\operatorname{dim} N /[N, N]$, are natural coordinates on $N /[N, N]$ corresponding to the simple roots and $\psi: \mathbb{F}_{q} \rightarrow \overline{\mathbb{Q}}_{\ell}^{\times}$is a fixed non-trivial character.

Consider the space $\overline{\mathbb{Q}}_{\ell}(G / K)_{\Psi}^{N}$ of left $(N, \Psi)$-equivariant and right $K$-invariant functions on $G$ that have a compact support modulo $N$. This space is a module over $\mathcal{H}(G, K)$, with the action defined by formula (5.1). For $\lambda \in{ }^{L} P^{+}$, let $\phi_{\lambda}$ be the function from $\overline{\mathbb{Q}}_{\ell}(G / K)_{\Psi}^{N}$, which vanishes outside the $N$-orbit of $\lambda(\pi)$ and equals $q^{-(\lambda, \rho)}$ at $\lambda(\pi)$. The elements $\phi_{\lambda}, \lambda \in{ }^{L} P^{+}$, provide a $\overline{\mathbb{Q}}_{\ell}$-basis for $\overline{\mathbb{Q}}_{\ell}(G / K)_{\Psi}^{N}$.

Define the linear map $\Phi: \overline{\mathbb{Q}}_{\ell}(G / K)^{K} \rightarrow \overline{\mathbb{Q}}_{\ell}(G / K)_{\Psi}^{N}$ by the formula

$$
(\Phi(f))(g)=\int_{N} f(u g) \Psi^{-1}(u) d u,
$$

where $d u$ stands for the Haar measure on $N$ normalized so that $\int_{N(\mathcal{O})} d u=1$.

Lemma 5.1. The map $\Phi$ defines an isomorphism of $\mathcal{H}$-modules

$$
\overline{\mathbb{Q}}_{\ell}(G / K)^{K} \simeq \overline{\mathbb{Q}}_{\ell}(G / K)_{\Psi}^{N} .
$$

Proof. Let $c_{\lambda} \in \overline{\mathbb{Q}}_{\ell}(G / K)^{K}$ be the characteristic function of the $K$-orbit of the $\operatorname{coset} \lambda(\pi) \cdot K \in G / K$. It follows from the definition that

$$
\Phi\left(c_{\lambda}\right)=q^{(\lambda, \rho)} \phi_{\lambda}+\sum_{\mu \in{ }^{L} P^{+} ; \mu<\lambda} b_{\lambda, \mu} \phi_{\mu} .
$$

This implies the lemma.

It is natural to call $\Phi$ the Fourier transform. We are now ready to state our main local theorem.

Theorem 5.2. The map $\Phi$ sends $H_{\lambda}$ to $\phi_{\lambda}$.

This theorem is equivalent to the formula

$$
\int_{N} H_{\lambda}(u \cdot \nu(\pi)) \Psi^{-1}(u) d u=q^{-(\lambda, \rho)} \delta_{\lambda, \nu} .
$$


5.4. Proof of Theorem 5.2. Our proof relies on the result of Casselman-Shalika (and Shintani for $G=G L_{n}$ ), which describes the values of the Whittaker function at the points $\mu(\pi)$ (cf. Theorem 2.1 and Remark 2.1).

Let $\gamma$ be a semi-simple conjugacy class in ${ }^{L} G$ and let $W_{\gamma}$ be a $\overline{\mathbb{Q}}_{\ell}$-valued function on $G$ with the following three properties:

- $W_{\gamma}(g h)=W_{\gamma}(g), \forall h \in K, W_{\gamma}(1)=1$;

- $W_{\gamma}(u g)=\Psi^{-1}(u) W_{\gamma}(g), \forall u \in N$;

$$
\int_{G} f(x) W_{\gamma}(g x) d x=\chi_{\gamma}(f) W_{\gamma}(g), \quad \forall g \in G, f \in \mathcal{H}
$$

(see Remark 5.1 for the definition of $\chi_{\gamma}$ ).

Theorem 5.3 ([5], [33]). The function $W_{\gamma}$ satisfying these properties exists, and it is unique. For $\mu \in{ }^{L} P$, the value of this function at $\mu(\pi)$ is

$$
W_{\gamma}(\mu(\pi))=q^{-(\mu, \rho)} \operatorname{Tr}(\gamma, V(\mu))
$$

if $\mu$ is a dominant weight, and 0 otherwise.

The function $W_{\gamma}$ is called the Whittaker function corresponding to $\gamma$.

Now we can prove Theorem 5.2. Let $\gamma$ be as above and let $s_{\gamma}$ be a linear functional $\overline{\mathbb{Q}}_{\ell}(G / K)_{\Psi}^{N} \rightarrow \overline{\mathbb{Q}}_{\ell}$ given by the formula

$$
s_{\gamma}(\phi)=\int_{N \backslash G} W_{\gamma}(g) \phi(g) d g,
$$

where $d g$ is the measure on $N \backslash G$ induced by the Haar measure on $G$ from Sect. 5.1 and the Haar measure on $N$ from Sect. 5.3. By construction, the function $W_{\gamma}(u) \phi(u)$ is left $N$-invariant. The integral (5.7) converges, because, by definition, $\phi$ has compact support modulo $N$.

Lemma 5.2. The map $s_{\gamma}$ is a homomorphism of $\mathcal{H}(G, K)$-modules $\overline{\mathbb{Q}}_{\ell}(G / K)_{\Psi}^{N} \rightarrow$ $\overline{\mathbb{Q}}_{\ell, \gamma}$, where $\overline{\mathbb{Q}}_{\ell, \gamma}$ is the one-dimensional representation of $\mathcal{H}(G, K)$ corresponding to its character $\chi_{\gamma}$.

Proof. Each $f \in \mathcal{H}$ acts on $\overline{\mathbb{Q}}_{\ell}(G / K)_{\Psi}^{N}$ by mapping $\phi \in \overline{\mathbb{Q}}_{\ell}(G / K)_{\Psi}^{N}$ to $f \cdot \phi$. By definition of the convolution product (see formula (5.1)), we have:

$$
(f \cdot \phi)(y)=\int_{G} f(x) \phi\left(y x^{-1}\right) d x .
$$

Hence

$$
s_{\gamma}(f \cdot \phi)=\int_{N \backslash G} W_{\gamma}(g)\left(\int_{G} f(x) \phi\left(g x^{-1}\right) d x\right) d g .
$$

Changing the order of integration and using the invariance of the Haar measure, we obtain

$$
s_{\gamma}(f \cdot \phi)=\int_{N \backslash G}\left(\int_{G} W_{\gamma}(g x) f(x) d x\right) \phi(g) d g
$$

By (5.5),

$$
s_{\gamma}(f \cdot \phi)=\chi_{\gamma}(f) s_{\gamma}(\phi)
$$


By formula (5.6) and the definition of the function $\phi_{\lambda}$, the function $W_{\gamma} \cdot \phi_{\lambda}$ equals $q^{-2(\lambda, \rho)} \operatorname{Tr}(\gamma, V(\lambda))$ times the characteristic function of the double coset $N \lambda(\pi) K$. Hence $s_{\gamma}\left(\phi_{\lambda}\right)=\int_{N \backslash G} W_{\gamma}(g) \phi_{\lambda}(g) d g$ equals $q^{-(\lambda, \rho)} \operatorname{Tr}(\gamma, V(\lambda))$ times the measure of the right $K$-orbit $\lambda(\pi) \cdot K$ in $N \backslash G$. This measure equals $\mu\left(K / A d_{\lambda(\pi)}(N(\mathcal{O}))\right)=$ $\mu(N(\mathcal{O})) / \mu\left(A d_{\lambda(\pi)}(N(\mathcal{O}))\right)$ due to our normalization. The latter equals $q^{2(\lambda, \rho)}$. Therefore $s_{\gamma}\left(\phi_{\lambda}\right)=\operatorname{Tr}(\gamma, V(\lambda))$ for each $\lambda \in{ }^{L} P^{+}$.

Any $\phi \in \overline{\mathbb{Q}}_{\ell}(G / K)_{\Psi}^{N}$ can be written as a finite sum $\sum_{\lambda \in L^{L} P^{+}} a_{\lambda} \phi_{\lambda}$, where $a_{\lambda} \in$ $\overline{\mathbb{Q}}_{\ell}$. We can identify the vector space $\overline{\mathbb{Q}}_{\ell}(G / K)_{\Psi}^{N}$ with $\operatorname{Rep}^{L} G\left(\overline{\mathbb{Q}}_{\ell}\right)$, by mapping $\phi_{\lambda}$ to $[V(\lambda)]$. Let $\bar{\phi}$ be the image of $\phi$ in $\operatorname{Rep}^{L} G\left(\overline{\mathbb{Q}}_{\ell}\right)$ under this identification. Then $s_{\gamma}(\phi)=\sum_{\lambda \in L P^{+}} a_{\lambda} \operatorname{Tr}(\gamma, V(\lambda))$ is simply the value of $\bar{\phi}$ at $\gamma \in \operatorname{Spec} \operatorname{Rep}^{L} G\left(\overline{\mathbb{Q}}_{\ell}\right)$. Since the algebra $\operatorname{Rep}^{L} G\left(\overline{\mathbb{Q}}_{\ell}\right)$ has no nilpotents, $\phi=\phi^{\prime}$, if and only if $s_{\gamma}(\phi)=s_{\gamma}\left(\phi^{\prime}\right)$ for all semi-simple conjugacy classes $\gamma$ in ${ }^{L} G\left(\overline{\mathbb{Q}}_{\ell}\right)$,

Therefore to prove Theorem 5.2, it is sufficient to check that for each semi-simple conjugacy class $\gamma$ in ${ }^{L} G\left(\overline{\mathbb{Q}}_{\ell}\right)$ and $\lambda \in{ }^{L} P^{+}$,

$$
s_{\gamma} \circ \Phi\left(H_{\lambda}\right)=\operatorname{Tr}(\gamma, V(\lambda)) .
$$

But the composition $s_{\gamma} \circ \Phi: \overline{\mathbb{Q}}_{\ell}(G / K)^{K} \rightarrow \overline{\mathbb{Q}}_{\ell, \gamma}$ is a homomorphism of $\mathcal{H}$-modules, by Lemma 5.1 and Lemma 5.2 . It is easy to check directly that the value of this homomorphism on the element $H_{0}=\operatorname{ch}_{K} \in \overline{\mathbb{Q}}_{\ell}(G / K)^{K}$ equals 1 . Therefore

$$
s_{\gamma} \circ \Phi\left(H_{\lambda}\right)=s_{\gamma} \circ \Phi\left(H_{\lambda} \cdot H_{0}\right)=\chi_{\gamma}\left(H_{\lambda}\right) \cdot s_{\gamma} \circ \Phi\left(H_{0}\right)=\chi_{\gamma}\left(H_{\lambda}\right)=\operatorname{Tr}(\gamma, V(\lambda))
$$

(see Remark 5.1), and Theorem 5.2 follows.

Remark 5.2. Our proof shows that Theorem 5.2 is equivalent to Theorem 5.3.

5.5. Proof of Proposition 4.2. Note that $(-1)^{2(\lambda, \rho)}=(-1)^{|\lambda|(n-1)}$. Hence we obtain from Proposition 5.1 and formula (4.9):

$$
L_{E, x}=\sum_{\lambda \in P_{n}^{++}} q_{x}^{(\lambda, \rho)+n(\lambda)} \operatorname{Tr}\left(\sigma\left(\operatorname{Fr}_{x}\right), E_{x}(\lambda)\right) \cdot H_{\lambda} .
$$

Therefore we find:

$$
\begin{aligned}
\int_{N^{J}\left(\mathcal{K}_{x}\right)} & L_{E, x}\left(u_{x} \cdot \operatorname{diag}\left(\pi_{x}^{\nu_{1}}, \ldots, \pi_{x}^{\nu_{n}}\right)\right) \Psi^{-1}\left(u_{x}\right) d u_{x} \\
= & \sum_{\lambda \in P_{n}^{++}} q_{x}^{(\lambda, \rho)+n(\lambda)} \operatorname{Tr}\left(\sigma\left(\operatorname{Fr}_{x}\right), E_{x}(\lambda)\right) \\
& \cdot \int_{N^{J}\left(\mathcal{K}_{x}\right)} H_{\lambda}\left(u_{x} \cdot \operatorname{diag}\left(\pi_{x}^{\nu_{1}}, \ldots, \pi_{x}^{\nu_{n}}\right)\right) \Psi^{-1}\left(u_{x}\right) d x .
\end{aligned}
$$

According to (5.4), the latter sum equals $q_{x}^{n(\nu)} \operatorname{Tr}\left(\sigma\left(\operatorname{Fr}_{x}\right), E_{x}(\nu)\right)$, which is the right hand side of formula (4.5). Now Proposition 4.2 is proved, and this finishes the proof of Proposition 3.2 and Theorem 3.1.

\section{WhitTAKER FUNCTIONS AND SPHERICAL FUNCTIONS}

In this section we give an interpretation of Theorem 5.2 from the point of view of the theory of spherical functions. Throughout this section we will work over the field of complex numbers instead of $\overline{\mathbb{Q}}_{\ell}$. In particular, all functions will be $\mathbb{C}$-valued, and $\mathcal{H}$ will be a $\mathbb{C}$-algebra. 
6.1. The map $\Theta$. Denote by $C^{\infty}(G / K)^{K}$ (resp., $\left.C^{\infty}(G / K)_{\Psi}^{N}\right)$ the space of smooth left $K$-invariant (resp., $(N, \Psi)$-equivariant) and right $K$-invariant functions on $G$. We also denote by $\mathbb{C}(G / K)^{K}$ (resp., $\mathbb{C}(G / K)_{\Psi}^{N}$ ) the subspace of compactly supported (resp., compactly supported modulo $N$ ) functions.

Each element of $C^{\infty}(G / K)^{K}$ can be written as an infinite sum $\sum_{\lambda \in{ }^{L} P^{+}} a_{\lambda} c_{\lambda}$, where $c_{\lambda}$ is the characteristic function of the $G(\mathcal{O})$-orbit $\operatorname{Gr}(G)^{\lambda}$.

Lemma 6.1. For each $g \in G,\left(\Phi\left(c_{\lambda}\right)\right)(g)=0$ for all but finitely many $\lambda \in{ }^{L} P^{+}$.

Proof. It suffices to prove the statement for $g=\mu(\pi)$. In this case, it is easy to see that for all but finitely many $\lambda$, there exists an element $v \in N$ (depending on $\lambda$ ) with $\Psi(v) \neq 1$, such that $\forall u \in N, u \cdot \mu(\pi) \in G r(G)^{\lambda}$ if and only if $(v u) \cdot \mu(\pi) \in G r(G)^{\lambda}$. But then $\left(\Phi\left(c_{\lambda}\right)\right)(\mu(\pi))=\Psi(v)\left(\Phi\left(c_{\lambda}\right)\right)(\mu(\pi))$, and hence $\left(\Phi\left(c_{\lambda}\right)\right)(g)=0$.

Therefore $\Phi$ defines a map $C^{\infty}(G / K)^{K} \rightarrow C^{\infty}(G / K)_{\Psi}^{N}, f \rightarrow \Phi(f)$, which is equivariant with respect to the action of Hecke operators.

Now we define a map $\Theta: C^{\infty}(G / K)_{\Psi}^{N} \rightarrow C^{\infty}(G / K)^{K}$ by the formula

$$
(\Theta(f))(g)=\int_{K} f(k g) d k
$$

where $d k$ stands for the Haar measure on $K$ of volume 1 . This map is also equivariant with respect to the action of Hecke operators.

We define $a$ as the element of $\mathbb{C}(G / K)^{K}$ equal to $(\Theta \circ \Phi)\left(\operatorname{ch}_{K}\right)=\Theta\left(\phi_{0}\right)$. The same argument as in the proof of Lemma 6.1 shows that the map $\Theta$ sends functions from $\mathbb{C}(G / K)_{\Psi}^{N}$ to $\mathbb{C}(G / K)^{K}$. Hence $a \in \mathbb{C}(G / K)^{K}=\mathcal{H}$.

Introduce the notation

$$
(a * f)(g)=\int_{G} a(x) f(g x) d x .
$$

Then we obtain:

$$
(\Theta \circ \Phi)(f)=a * f, \quad \forall f \in C^{\infty}(G / K)^{K} .
$$

In the next section we will use the element $a$ to clarify the connection between Whittaker functions and spherical functions.

6.2. Connection between $a$ and the Plancherel measure. Let $\gamma$ be a semisimple conjugacy class in the group ${ }^{L} G(\mathbb{C})$. Recall [31], [28] that the spherical function $S_{\gamma}$ is the unique $K$-bi-invariant function on $G$, such that

- $f * S_{\gamma}=\chi_{\gamma}(f) S_{\gamma}, \forall f \in \mathcal{H}$, where $\chi_{\gamma}: \mathcal{H} \rightarrow \mathbb{C}$ is the character corresponding to $\gamma$ defined in Remark 5.1;

- $S_{\gamma}(1)=1$.

These properties imply that

$$
\int_{G} f(x) S_{\gamma}(x) d x=\chi_{\gamma}(f) .
$$

Now let $W_{\gamma}$ be the Whittaker function on $G$ as defined in Sect. 5.4 but with the character $\Psi^{-1}$ of $N$ replaced with the character $\Psi$. It is straightforward to check that the function $\Phi\left(S_{\gamma}\right)$ satisfies all the properties of the function $W_{\gamma}$ from Sect. 5.4, except for the normalization condition $W_{\gamma}(1)=1$. By Theorem 5.3, $\Phi\left(S_{\gamma}\right)$ is proportional to $W_{\gamma}$. 


\section{Lemma 6.2.}

$$
\Phi\left(S_{\gamma}\right)=\chi_{\gamma}(a) W_{\gamma}
$$

Proof. Introduce $a(\gamma)$ by the formula $\Phi\left(S_{\gamma}\right)=a(\gamma) W_{\gamma}$. Since $\Theta\left(W_{\gamma}\right)=S_{\gamma}$ by definition, we obtain, using formula (6.2) and the properties of $S_{\gamma}: a(\gamma) S_{\gamma}=$ $(\Theta \circ \Phi)\left(S_{\gamma}\right)=a * S_{\gamma}=\chi_{\gamma}(a) S_{\gamma}$.

According to [28], (1.5.1), there exists a unimodular measure $d \mu(\gamma)$ (Plancherel measure) on the maximal compact subtorus ${ }^{L} T^{u}$ of ${ }^{L} T$, which satisfies

$$
\int_{G} f_{1}(g) \overline{f_{2}(g)} d g=\int_{L_{T^{u}}} \chi_{\gamma}\left(f_{1}\right) \overline{\chi_{\gamma}\left(f_{2}\right)} d \mu(\gamma),
$$

for all $f_{1}, f_{2} \in \mathcal{H}$.

Setting $f_{2}=\operatorname{ch}_{K}$, we obtain:

$$
f(1)=\int_{L_{T^{u}}} \chi_{\gamma}(f) d \mu(\gamma), \quad \forall f \in \mathcal{H} .
$$

By Theorem 5.2, $\Phi\left(H_{\lambda}\right)=\phi_{\lambda}$. But it is clear that $\left(\Theta\left(\phi_{\lambda}\right)\right)(1)=\delta_{\lambda, 0}$. Therefore, using (6.2), we see that $\left(a * H_{\lambda}\right)(1)=\delta_{\lambda, 0}$. Substituting this into formula (6.5) and using the formula $\chi_{\gamma}\left(H_{\lambda}\right)=\operatorname{Tr}(\gamma, V(\lambda))$, we obtain:

$$
\int_{L_{T^{u}}} \operatorname{Tr}(\gamma, V(\lambda)) a(\gamma) d \mu(\gamma)=\delta_{\lambda, 0} .
$$

There exists a unique measure $d \widetilde{\mu}(\gamma)$ on ${ }^{L} T^{u}$ (induced by the Haar measure on $\left.{ }^{L} G^{u}\right)$, such that

$$
\int_{L_{T^{u}}} \operatorname{Tr}(\gamma, V(\nu)) \overline{\operatorname{Tr}(\gamma, V(\lambda))} d \widetilde{\mu}(\gamma)=\delta_{\lambda, \nu}
$$

Formula (6.6) then implies

\section{Proposition 6.1.}

$$
a(\gamma)=\frac{d \widetilde{\mu}(\gamma)}{d \mu(\gamma)}
$$

Now Lemma 6.2 gives us:

$$
\Phi\left(S_{\gamma}\right)=\frac{d \widetilde{\mu}(\gamma)}{d \mu(\gamma)} W_{\gamma}
$$

6.3. Another proof of Theorem 5.2. In this subsection, which is independent from the previous one, we use spherical functions to give another proof of Theorem 5.2.

Substituting $f_{1}=\operatorname{ch}_{K g K}$ into formula (6.4) and using formula (6.3), we obtain that for any $f \in \mathcal{H}$,

$$
\overline{f(g)}=\int_{L_{T^{u}}} S_{\gamma}(g) \overline{\chi_{\gamma}(f)} d \mu(\gamma) .
$$

Since $\chi_{\gamma}\left(H_{\lambda}\right)=\operatorname{Tr}(\gamma, V(\lambda))$, we have:

$$
H_{\lambda}(g)=\int_{L_{T^{u}}} S_{\gamma}(g) \overline{\operatorname{Tr}(\gamma, V(\lambda))} d \mu(\gamma) .
$$


According to formula (6.10),

$$
H_{0}(g)=\int_{L_{T^{u}}} S_{\gamma}(g) d \mu(\gamma)
$$

Hence

$$
\Phi\left(H_{0}\right)(g)=\int_{L_{T^{u}}} \Phi\left(S_{\gamma}\right)(g) d \mu(\gamma)=\int_{L_{T^{u}}} W_{\gamma}(g) a(\gamma) d \mu(\gamma)
$$

On the other hand, it is clear from definition that $\Phi\left(H_{0}\right)=\phi_{0}$. Therefore, substituting $g=\lambda(\pi)$ and using formula (5.6), we obtain formula (6.6). Repeating the argument with the Haar measure given above, we obtain (6.8).

Now formulas (6.10), (6.8) and (5.6) give:

$$
\begin{gathered}
\left(\Phi\left(H_{\lambda}\right)\right)(\nu(\pi))=\int_{{ }^{T^{u}}} W_{\gamma}(\nu(\pi)) \overline{\operatorname{Tr}(\gamma, V(\lambda))} d \widetilde{\mu}(\gamma) \\
=q^{-(\nu, \rho)} \int_{L_{T^{u}}} \operatorname{Tr}(\gamma, V(\nu)) \overline{\operatorname{Tr}(\gamma, V(\lambda))} d \widetilde{\mu}(\gamma)=q^{-(\lambda, \rho)} \delta_{\lambda, \nu} .
\end{gathered}
$$

This proves formula (5.4) and Theorem 5.2 over the field of complex numbers. Since $H_{\lambda}$ takes values in rational numbers and $\Psi$ takes values in the roots of unity, the validity of (5.4) over $\mathbb{C}$ implies its validity over $\overline{\mathbb{Q}}_{\ell}$.

6.4. The function $L_{\gamma}$. The Whittaker function can be written as a series

$$
W_{\gamma}=\sum_{\lambda \in{ }^{L} P^{+}} \operatorname{Tr}(\gamma, V(\lambda)) \cdot \phi_{\lambda} .
$$

This series obviously makes sense, since the supports of the functions $\phi_{\lambda}$ do not intersect. In view of Theorem 5.2, it is natural to consider the series

$$
L_{\gamma}=\sum_{\lambda \in{ }^{L} P^{+}} \operatorname{Tr}(\gamma, V(\lambda)) \cdot H_{\lambda} .
$$

However, the convergence of this series is not at all automatic, because the supports of functions $H_{\lambda}$ do intersect; for instance, each $H_{\lambda}$ has a non-zero value at 1 . In this section we study the question of convergence of $L_{\gamma}$.

Let us write:

$$
H_{\lambda}=q^{-(\lambda, \rho)} \sum_{\mu \leq \lambda} P_{\mu \lambda}\left(q^{-1}\right) \cdot c_{\mu},
$$

where $q^{-(\lambda, \rho)} P_{\mu \lambda}$ is a polynomial in $q^{-1}$ (recall that $c_{\lambda}$ is the characteristic function of the $G(\mathcal{O})$-orbit $\left.\operatorname{Gr}(G)^{\lambda}\right)$. Formula (6.10) can be rewritten as follows:

$$
H_{\lambda}(g)=\int_{L_{T^{u}}} S_{\gamma}(g) a(\gamma)^{-1} \overline{\operatorname{Tr}(\gamma, V(\lambda))} d \widetilde{\mu}(\gamma) .
$$

Using the defining properties of the spherical function $S_{\gamma}$, we can write it as a series

$$
S_{\gamma}=\sum_{\mu \in{ }^{L} P^{+}} s_{\gamma}^{\mu}\left(q^{-1}\right) \cdot c_{\mu},
$$

where $s_{\gamma}^{\mu}\left(q^{-1}\right)$ is a rational function in $q^{-1}$ of the form $Q\left(q^{-1}\right) \widetilde{s}_{\gamma}^{\mu}\left(q^{-1}\right)$. Here

$$
Q\left(q^{-1}\right)=\prod_{i=1}^{l} \frac{1-q^{-m_{i}-1}}{1-q^{-1}}
$$


( $l$ is the rank of $G$, the $m_{i}$ 's are the exponents of $G$; note that $Q(q)=\# G / B\left(\mathbb{F}_{q}\right)$ ), and $\widetilde{s}_{\gamma}^{\mu}$ is a polynomial in $q^{ \pm 1}$. Its coefficients are finite integral linear combinations of characters of irreducible representations of ${ }^{L} G$ (for an explicit formula, see [28]). It follows from formula (6.2) that $a(\gamma)$ has the same structure as a function of $q^{-1}$. Hence both $s_{\gamma}^{\mu}\left(q^{-1}\right)$ and $a(\gamma)^{-1}$ can be viewed as formal Laurent power series in $q^{-1}$ and formula (6.13) can be viewed as an identity on such power series.

We have:

$$
s_{\gamma}^{\mu}=\sum_{m>-M} \operatorname{Tr}\left(\gamma, R_{m}^{\mu}\right) q^{-m}
$$

and

$$
a(\gamma)^{-1}=\sum_{m>-M^{\prime}} \operatorname{Tr}\left(\gamma, U_{m}\right) q^{-m},
$$

where $R_{m}^{\mu}$ and $U_{m}$ are finite linear combinations of irreducible representations of ${ }^{L} G$ (the summation is actually only over $m \in \mathbb{Z}_{+}$). Then formula (6.13) can be written as follows:

$$
q^{-(\lambda, \rho)} P_{\mu \lambda}\left(q^{-1}\right)=\sum_{N \in \mathbb{Z}} q^{-N} \int_{{ }^{L} T^{u}} \operatorname{Tr}\left(\gamma, \bigoplus_{m \in \mathbb{Z}} R_{m}^{\mu} \otimes U_{N-m}\right) \overline{\operatorname{Tr}(\gamma, V(\lambda))} d \widetilde{\mu}(\gamma) .
$$

By formula (6.7), the $q^{-N}$ coefficient of $P_{\mu \lambda}\left(q^{-1}\right)$ equals the multiplicity of $V(\lambda)$ in $\bigoplus_{m \in \mathbb{Z}} R_{m}^{\mu} \otimes U_{N-m}$. But by construction the latter is a finite linear combination of irreducible representations of ${ }^{L} G$. Hence we obtain the following

Lemma 6.3. For each $\mu \in{ }^{L} P^{+}$and $N \in \mathbb{Z}_{+}$there are only finitely many $\lambda \in$ ${ }^{L} P^{+}$, such that $q^{-(\lambda, \rho)} P_{\mu \lambda}$ has a non-zero coefficient in front of $q^{-N}$.

Remark 6.1. This can also be seen from the explicit formula for $P_{\mu \lambda}$ obtained in [27], [17].

Therefore for each $g \in G, L_{\gamma}(g)$ given by formula (6.12) makes sense as a formal power series in $q^{-1}$, each coefficient being a finite linear combination of characters. Furthermore, we see, by reversing the argument above that as formal power series in $q^{-1}$,

$$
L_{\gamma}(g)=a(\gamma)^{-1} S_{\gamma}(g), \quad \forall g \in G .
$$

In order to estimate the convergence of this series, we have to compute $a(\gamma)$ explicitly. According to [28], (5.1.2),

$$
d \mu(\gamma)=\frac{Q\left(q^{-1}\right)}{|W|} \frac{\prod_{\alpha \in \Delta}(1-\alpha(\gamma))}{\prod_{\alpha \in \Delta}\left(1-q^{-1} \alpha(\gamma)\right)} d \gamma,
$$

where $|W|$ is the number of elements in the Weyl group, and $\Delta$ is the set of roots of $G$. The notation $d \gamma$ means the Haar measure on ${ }^{L} T^{u}$, which gives it volume 1 . On the other hand,

$$
d \widetilde{\mu}(\gamma)=\frac{1}{|W|} \prod_{\alpha \in \Delta}(1-\alpha(\gamma)) d \gamma
$$

Now Proposition 6.1 gives:

$$
a(\gamma)=\frac{\prod_{\alpha \in \Delta}\left(1-q^{-1} \alpha(\gamma)\right)}{Q\left(q^{-1}\right)} .
$$


Let ${ }^{L} \mathfrak{g}$ be the adjoint representation of ${ }^{L} G$, and $\Lambda^{i}\left({ }_{\mathfrak{g}}\right)$ be its $i$ th exterior power. Formula (6.17) means that $a \in \mathcal{H}$ is the image under the Satake isomorphism $S$ of the following element in $\operatorname{Rep}^{L} G$ :

$$
\prod_{i=1}^{l}\left(1-q^{-m_{i}-1}\right)^{-1} \sum_{i=0}^{\operatorname{dim}^{L} \mathfrak{g}}\left[\Lambda^{i}\left({ }_{\mathfrak{g}}\right)\right](-1)^{i} q^{-i} .
$$

Formula (6.16) implies the following result.

Proposition 6.2. If the conjugacy class $\gamma$ satisfies: $q^{-1}<|\alpha(\gamma)|<q, \forall \alpha \in \Delta_{+}$, then $L_{\gamma}(g)$ converges absolutely to

$$
\frac{Q\left(q^{-1}\right)}{\prod_{\alpha \in \Delta}\left(1-q^{-1} \alpha(\gamma)\right)} S_{\gamma}(g)
$$

for all $g \in G$.

Note that when $G=G L_{n}$, formula (6.12) looks similar to formula (5.8) for the function $L_{E, x}$. Besides powers of $q_{x}$, the difference is that in (5.8) the summation is restricted to the subset $P_{n}^{++}$of the set $P_{n}^{+}={ }^{L} P^{+}$of all dominant weights of $G L_{n}$. However, $L_{E, x}$ is not the restriction of (6.12) to the union of strata $\operatorname{Gr}\left(G L_{n}\right)^{\lambda}$ with $\lambda \in P_{n}^{++}$, because the functions $H_{\lambda}$ with $\lambda \in P_{n}^{+}-P_{n}^{++}$do not vanish on those strata. While $L_{\gamma}$ given by (6.12) is manifestly an eigenfunction of the Hecke operators, $L_{E, x}$ is not. ${ }^{3}$

For general $G$ there is no analogue of the subset ${ }^{L} P^{++} \subset{ }^{L} P^{+}$, and so the function $L_{\gamma}$ seems to be the closest analogue of $L_{E, x}$ in the general setting. According to Theorem 5.2 and formula $(5.6), \Phi\left(L_{\gamma}\right)$ equals the Whittaker function $W_{\gamma}$.

Remark 6.2. Let $\gamma$ be a semi-simple conjugacy class of ${ }^{L} G\left(\overline{\mathbb{Q}}_{\ell}\right)$ and $r:{ }^{L} G\left(\overline{\mathbb{Q}}_{\ell}\right) \rightarrow$ Aut $V$ a finite-dimensional representation of ${ }^{L} G\left(\overline{\mathbb{Q}}_{\ell}\right)$. Recall that the local $L-$ function associated to the pair $(\gamma, V)$ is defined by the formula

$$
L(\gamma, V ; s)=\operatorname{det}\left(1-r(\gamma) q^{-s}\right)^{-1} \text {. }
$$

In particular, if $V=L_{\mathfrak{g}}$ is the adjoint representation, then

$$
L\left(\gamma,{ }^{L} \mathfrak{g} ; s\right)=\left(1-q^{-s}\right)^{-l} \prod_{\alpha \in \Delta}\left(1-\alpha(\gamma) q^{-s}\right)^{-1} .
$$

Hence $a(\gamma)$ can be written as

$$
a(\gamma)=L\left(\gamma,{ }^{L} \mathfrak{g} ; 1\right) \prod_{i=1}^{l}\left(1-q^{-m_{i}-1}\right) .
$$

Thus, we obtain:

$$
\Phi\left(S_{\gamma}\right)=L\left(\gamma,{ }^{L} \mathfrak{g} ; 1\right)^{-1} \prod_{i=1}^{l}\left(1-q^{-m_{i}-1}\right)^{-1} \cdot W_{\gamma} .
$$

Using arguments similar to those of Casselman and Shalika [5], one can show that the irreducible unramified representation corresponding to $\gamma$ has a Whittaker model if and only if $\Phi\left(S_{\gamma}\right) \neq 0$. Formula (6.19) means that $\Phi\left(S_{\gamma}\right) \neq 0$ if and only if $L\left(\gamma,{ }^{L} \mathfrak{g} ; s\right)$ is regular at $s=1$. We conclude that the irreducible unramified

\footnotetext{
${ }^{3}$ It is actually an eigenfunction of some other operators, similar to the Hecke operators, which were defined by Laumon [22].
} 
representation of $G$ with the Langlands parameter $\gamma$ has a Whittaker model if and only if $L\left(\gamma,{ }^{L} \mathfrak{g} ; s\right)$ is regular at $s=1$ (in that case the Whittaker model is actually unique). This agrees with a special case of a conjecture of Gross and Prasad [12] (Conjecture 2.6).

6.5. Identities on $P_{\mu \lambda}$. According to formula (6.16), for each $\mu \in{ }^{L} P^{+}$we have the following equality of power series in $q^{-1}$ :

$$
\sum_{\lambda: \lambda \geq \mu} \operatorname{Tr}(\gamma, V(\lambda)) q^{-(\lambda, \rho)} P_{\mu \lambda}\left(q^{-1}\right)=\prod_{i=1}^{l} \frac{1-q^{-m_{i}-1}}{1-q^{-1}} \prod_{\alpha \in \Delta}\left(1-q^{-1} \alpha(\gamma)\right)^{-1} S_{\gamma}(\mu(\pi)) .
$$

Recall that the coefficients of the polynomial $P_{\mu \lambda}$ (which can be interpreted as a Kazhdan-Lusztig polynomial for the affine Weyl group [27], [17]) are given by dimensions of stalk cohomologies of the perverse sheaf $\mathcal{A}_{\lambda}$ :

$$
P_{\mu \lambda}=\left.q^{(\lambda, \rho)} \sum_{i \in \mathbb{Z}} \operatorname{dim} \mathrm{H}^{i}\left(\mathcal{A}_{\lambda}\right)\right|_{\mu(\pi)} q^{i / 2} .
$$

Thus, formula (6.20) is an identity which connects these dimensions with the values of the spherical functions. The latter are known explicitly; they can be expressed via the Hall-Littlewood polynomials [28].

For example, let us apply formula (6.20) when $G=S L_{2}$ and $\mu=0$. In this case, $\gamma \in \mathbb{C}^{\times}$, and the set ${ }^{L} P^{+}$of dominant weights of the dual group ${ }^{L} G=P G L_{2}$ can be identified with the set of non-negative even integers. To weight $2 m$ corresponds the $2 m+1$-dimensional representation $V(2 m)$ of $P G L_{2}$, and $\operatorname{Tr}(\gamma, V(2 m))=\sum_{i=-m}^{m} \gamma^{i}$. Formula (6.20) then gives:

$$
\left.\sum_{m \in \mathbb{Z}_{+}}\left(\sum_{j=-m}^{m} \gamma^{j}\right) \cdot \sum_{i \in \mathbb{Z}} \operatorname{dim} H^{i}\left(\mathcal{A}_{2 m}\right)\right|_{1} q^{i / 2}=\frac{1+q^{-1}}{\left(1-q^{-1} \gamma\right)\left(1-q^{-1} \gamma^{-1}\right)}
$$

This is easy to see directly, because $\mathcal{A}_{2 m}$ is known to be the constant sheaf on the closure of the stratum $\mathcal{G r}\left(S L_{2}\right)^{2 m}$ placed in degree $-2 m$.

For general $G$, formula (6.20) with $\mu=0$ can be interpreted as follows. Let $R\left({ }^{L} \mathfrak{g}\right)$ be the graded ring of polynomials on ${ }^{L} \mathfrak{g}, J\left({ }_{\mathfrak{g}}\right)$ be its subring of ${ }^{L} G$-invariants, and $H\left({ }^{L} \mathfrak{g}\right)$ be the subspace of ${ }^{L} G$-harmonic polynomials on ${ }^{L} \mathfrak{g}$.

For a graded space $V$, we denote by $V_{j}$ its $j$ th homogeneous component. If each $V_{j}$ is a representation of ${ }^{L} G$, we denote by $\operatorname{Ch}(\gamma, V)$ the graded character of $V$ :

$$
\operatorname{Ch}(\gamma, V)=\sum_{j=0}^{\infty} \operatorname{Tr}\left(\gamma, V_{j}\right) q^{-j}
$$

Note that the graded character of $R\left({ }^{L} \mathfrak{g}\right)$ equals

$$
\operatorname{Ch}\left(\gamma, R\left({ }^{L} \mathfrak{g}\right)\right)=\prod_{i=1}^{l}\left(1-q^{-1}\right)^{-l} \prod_{\alpha \in \Delta}\left(1-q^{-1} \alpha(\gamma)\right)^{-1}
$$

(compare with Remark 6.2), while

$$
\operatorname{Ch}\left(\gamma, J\left({ }^{L} \mathfrak{g}\right)\right)=\prod_{i=1}^{l}\left(1-q^{-m_{i}-1}\right)^{-1}
$$


Now let $H\left({ }_{\mathfrak{g}}\right)$ be the (graded) space of ${ }^{L} G$-harmonic polynomials on ${ }^{L} \mathfrak{g}$. According to Theorem 0.2 of B. Kostant [18], $R\left({ }_{\mathfrak{g}}\right)=J\left({ }^{L} \mathfrak{g}\right) \otimes H\left({ }^{L} \mathfrak{g}\right)$. Hence

$$
\operatorname{Ch}\left(\gamma, H\left({ }^{L} \mathfrak{g}\right)\right)=\prod_{i=1}^{l} \frac{1-q^{-m_{i}-1}}{1-q^{-1}} \prod_{\alpha \in \Delta}\left(1-q^{-1} \alpha(\gamma)\right)^{-1}=a(\gamma)^{-1} .
$$

Thus, we obtain another interpretation of $a(\gamma)^{-1}$ : it is equal to the graded character of the space of ${ }^{L} G$-harmonic polynomials. Note that it also equals the graded character of the ring of regular functions on the nilpotent cone $\mathcal{N}$ in ${ }^{L_{\mathfrak{g}}}$.

Formula (6.20) together with this interpretation give us the following result.

Proposition 6.3.

$$
P_{0, \lambda}\left(q^{-1}\right)=q^{(\lambda, \rho)} \sum_{j=0}^{\infty} q^{-j} \operatorname{mult}\left(V(\lambda), H\left({ }^{L} \mathfrak{g}\right)_{j}\right),
$$

where $\operatorname{mult}\left(V(\lambda), H\left({ }^{L_{\mathfrak{g}}}\right)_{j}\right)$ is the multiplicity of $V(\lambda)$ in $H\left({ }^{L_{\mathfrak{g}}}\right)_{j}$.

A complete description of these multiplicities has been given by Kostant in [18]. In fact, applying Theorem 0.11 of [18] to formula (6.23), we obtain:

$$
P_{0, \lambda}=q^{(\lambda, \rho)} \sum_{i=1}^{l_{\lambda}} q^{-m_{i}(\lambda)}
$$

where $m_{i}(\lambda)$ are the generalized exponents associated to the representation $V(\lambda)$, defined in [18]. In the special case when $V(\lambda)$ is the adjoint representation ${ }_{\mathfrak{g}}$, these are just the exponents of ${ }^{L} G$, and formula (6.24) specializes to Lusztig's formula (see [27], p. 226)

$$
P_{0, \lambda_{\text {adj }}}=\sum_{i=1}^{l} q^{m_{i}-1} .
$$

Formula (6.24) is not new: R. Brylinski [4] observed that it immediately follows if one compares the Lusztig-Kato formula [27], [17] for $P_{0, \lambda}$ and the HesselinkPeterson formula [14] for the right hand side of (6.24). Note that in contrast to her argument, our proof of formula (6.24) does not use the Lusztig-Kato formula.

Using (6.15) it is easy to derive a formula analogous to (6.23) for $P_{\mu \lambda}$ in terms of Hall-Littlewood polynomials.

\section{Geometric analogue of Theorem 5.2 and some open problems}

7.1. In this subsection we will formulate a geometric analogue of Theorem 5.2. Recall the Grassmannian $\operatorname{Gr}(G)$ of section 5.2. This is a strict ind-scheme over $\mathbb{F}_{q}$, i.e., an inductive system of $\mathbb{F}_{q}$-schemes $\mathcal{G r}(G)_{k}, k \geq 0$, where all maps $i_{k, m}$ : $\mathcal{G} r(G)_{k} \rightarrow \mathcal{G} r(G)_{m}, k<m$, are closed embeddings. For more details, see, e.g., [20], [29]. By a $\overline{\mathbb{Q}}_{\ell}$-sheaf on $\mathcal{G r}(G)$ we will understand a system of $\overline{\mathbb{Q}}_{\ell}$-sheaves $\mathcal{F}_{k}$ on each $\mathcal{G r}(G)_{k}$ and a compatible system of isomorphisms $\mathcal{F}_{k} \simeq i_{k, m}^{*} \mathcal{F}_{m}$ for all $k<m$.

There exists an ind-group scheme $\mathcal{N}(\mathcal{K})$, whose set of $\mathbb{F}_{q}$-points is $N(\mathcal{K})$. This ind-group scheme acts on the Grassmannian $\operatorname{Gr}(G)$, and its orbits stratify $\operatorname{Gr}(G)$ by ind-schemes $S^{\nu}, \nu \in{ }^{L} P$. The stratum $S^{\nu}$ is the $\mathcal{N}(\mathcal{K})$-orbit of $\nu(\pi) \in G r(G)$. Denote by $j^{\nu}$ the embedding $S^{\nu} \hookrightarrow \operatorname{Gr} r(G)$. 
We choose a generic additive character $\widetilde{\Psi}: \mathcal{N}(\mathcal{K}) \rightarrow \mathbb{G}_{a, \mathcal{K}}$ and define a homomorphism $\Psi: \mathcal{N}(\mathcal{K}) \rightarrow \mathbb{G}_{a, \mathbb{F}_{q}}$ by the formula

$$
\Psi=\operatorname{Res} \circ \widetilde{\Psi}: \mathcal{N}(\mathcal{K}) \rightarrow \mathbb{G}_{a, \mathbb{F}_{q}},
$$

where Res is the geometric analogue of the residue map of Sect. 5.3. As before, let $\psi: \mathbb{F}_{q} \rightarrow \overline{\mathbb{Q}}_{\ell}^{\times}$denote a non-trivial character and let $\mathcal{J}_{\psi}$ denote the corresponding Artin-Schreier sheaf on $\mathbb{G}_{a, \mathbb{F}_{q}}$.

Consider the category $\mathrm{P}_{\mathcal{G}(\mathcal{O})}(\mathcal{G r} r(G))$ of $\mathcal{G}(\mathcal{O})$-equivariant perverse sheaves on $\operatorname{Gr}(G)$ with finite-dimensional support. This category is a geometric analogue of the Hecke algebra $\mathcal{H}=\overline{\mathbb{Q}}_{\ell}(G / K)^{K}$ (see Remark 7.1 below). Now we define an abelian category $\operatorname{Sh}_{\mathcal{N}(\mathcal{K})}^{\Psi}(\mathcal{G} r(G))$ (a geometric analogue of $\overline{\mathbb{Q}}_{\ell}(G / K)_{\Psi}^{N}$ ) and a collection of cohomology functors $\Phi^{i}: \mathrm{P}_{\mathcal{G}(\mathcal{O})}(\mathcal{G} r(G)) \rightarrow \operatorname{Sh}_{\mathcal{N}(\mathcal{K})}^{\Psi}(\mathcal{G} r(G))$, which are a geometric analogue of the map $\Phi$ of Sect. 5.3.

The objects of the category $\operatorname{Sh}_{\mathcal{N}(\mathcal{K})}^{\Psi}(\mathcal{G} r(G))$ are $\overline{\mathbb{Q}}_{\ell}$-sheaves $\mathcal{E}$ on $\mathcal{G} r(G)$ which satisfy the following conditions:

(1) $j^{\nu *} \mathcal{E}=0$ except for finitely many $\nu \in{ }^{L} P$;

(2) $t_{\nu}^{*} j^{\nu *} \mathcal{E} \otimes \varphi^{*} \mathcal{J}_{\psi^{-1}}$ are trivial local systems of finite rank for all $\nu$, where $t_{\nu}$ : $\mathcal{N}(\mathcal{K}) \rightarrow S^{\nu}$ is the map $u \mapsto u \cdot \nu(t)$.

Morphisms in this category are defined in an obvious way.

Lemma 7.1. If $\nu \in{ }^{L} P$ is not dominant, then for every $\left.\mathcal{E} \in \mathrm{Ob}_{\left(\mathrm{Sh}_{\mathcal{N}(\mathcal{K})}^{\Psi}\right.}^{\Psi} \operatorname{Gr}(G)\right)$, $j^{\nu *}(\mathcal{E})=0$.

The proof is analogous to the proof of the corresponding statement for functions. Thus, $\mathcal{E}$ not only satisfies property (1) above, but also satisfies the stronger property

$\left(1^{\prime}\right) j^{\nu^{*} \mathcal{E}}=0$ except for finitely many $\nu \in{ }^{L} P^{+}$.

Now we construct the functors $\Phi^{i}: \mathrm{P}_{\mathcal{G}(\mathcal{O})}(\mathcal{G} r(G)) \rightarrow \operatorname{Sh}_{\mathcal{N}(\mathcal{K})}^{\Psi}(\mathcal{G} r(G))$. Consider the sequence of maps:

$$
\mathcal{G} r \stackrel{a}{\longleftarrow} \mathcal{N}(\mathcal{K}) \times \mathcal{G} r \stackrel{q}{\longrightarrow} \mathcal{N}(\mathcal{K}) / \mathcal{N}(\mathcal{O}) \times \mathcal{G} r \stackrel{p}{\longrightarrow} \mathcal{G} r,
$$

where $a$ is given by acting with $\mathcal{N}(\mathcal{K})$ on $\mathcal{G} r$ and $p, q$ are projections. For $\mathcal{F} \in$ $\mathrm{P}_{\mathcal{G}(\mathcal{O})}(\mathcal{G r}(G))$ we then set:

$$
\Phi^{i}(\mathcal{F})=R^{i} p_{!}\left(\widetilde{\mathcal{F}} \otimes \varphi^{*} \mathcal{J}_{\psi}\right), \quad \text { with } \quad q^{*} \widetilde{\mathcal{F}}=a^{*} \mathcal{F} .
$$

To formulate the geometric analogue of Theorem 5.2, recall that for $\lambda \in{ }^{L} P^{+}$we denote by $\mathcal{A}_{\lambda}$ the Goresky-MacPherson extension of the sheaf $\overline{\mathbb{Q}}_{\ell, \lambda}[2(\lambda, \rho)]((\lambda, \rho))$ associated to the $\mathcal{G}(\mathcal{O})$-orbit $\mathcal{G} r(G)^{\lambda}$. For each $\nu \in{ }^{L} P^{+}$, denote by $\mathcal{N}(\mathcal{K})_{\nu}$ the isotropy group of $\nu(\pi)$. Since $\nu$ is dominant, the restriction of $\Psi$ to $\mathcal{N}(\mathcal{K})_{\nu}$ equals 0 . Therefore the map $\Psi$ restricts to a map on $S^{\nu}$, which we continue to denote by the same letter $\Psi: S^{\nu} \rightarrow \mathbb{G}_{a, \mathbb{F}_{q}}$. With this notation, the sheaf $\Psi^{*} \mathcal{J}_{\psi}$ is a sheaf on $S^{\nu}$. Recall that $j^{\nu}$ denotes the embedding $S^{\nu} \hookrightarrow \operatorname{Gr}(G)$. Now we are ready to state the geometric analogue of Theorem 5.2.

\section{Conjecture 7.1.}

$$
\Phi^{i}\left(\mathcal{A}_{\lambda}\right)=\left\{\begin{array}{l}
j_{!}^{\lambda} \Psi^{*} \mathcal{J}_{\psi}((\lambda, \rho)) \quad \text { if } i=2(\lambda, \rho), \\
0 \quad \text { if } i \neq 2(\lambda, \rho) .
\end{array}\right.
$$

We will now formulate Conjecture 7.2 describing the stalk cohomologies of the sheaves $\Phi^{i}\left(\mathcal{A}_{\lambda}\right)$. The statement of Conjecture 7.2 does not explicitly involve the 
category $\operatorname{Sh}_{\mathcal{N}(\mathcal{K})}^{\Psi}(\mathcal{G r}(G))$ and the functors $\Phi^{i}$. However, Conjecture 7.1 can be derived from Conjecture 7.2.

Note that the support of the restriction of $\mathcal{A}_{\lambda}$ to $S^{\nu}$, i.e., $\overline{\mathcal{G r} r(G)^{\lambda}} \cap S^{\nu}$, is finitedimensional. To work in a geometric setting, let us extend the base field from $\mathbb{F}_{q}$ to $\overline{\mathbb{F}}_{q}$ and use Weil sheaves. Denote by $\Psi_{\lambda, \nu}$ the restriction of $\Psi$ to $\overline{\mathcal{G r}(G)^{\lambda}} \cap S^{\nu}$.

Conjecture 7.2. For $\lambda$ dominant

$$
\left.\mathrm{H}_{c}^{k} \overline{\left(\mathcal{G r}(G)^{\lambda}\right.} \cap S^{\nu}, \mathcal{A}_{\lambda} \otimes \Psi_{\lambda, \nu}^{*} \mathcal{J}_{\psi}\right)= \begin{cases}\overline{\mathbb{Q}}_{\ell}(-(\lambda, \rho)) & \text { if } \nu=\lambda \text { and } k=2(\lambda, \rho), \\ 0 & \text { otherwise. }\end{cases}
$$

Proving Conjecture 7.2 would yield an alternative proof of Theorem 5.2, and hence of the Casselman-Shalika formula (5.6) (see Remark 5.2).

One sees readily that Conjecture 7.2 holds when $\lambda \leq \nu$. Let us, then, assume that $\nu$ is dominant and $\nu<\lambda$. The projection formula implies that

$$
\mathrm{H}_{c}^{*}\left(S^{\nu}, \mathcal{A}_{\lambda} \otimes \Psi^{*} \mathcal{J}_{\psi}\right)=\mathrm{H}_{c}^{*}\left(\mathbb{G}_{a, \overline{\mathbb{F}}_{q}}, R \Psi_{!} \mathcal{A}_{\lambda} \otimes \mathcal{J}_{\psi}\right) .
$$

Therefore Conjecture 7.2 follows from the following

Conjecture 7.3. For $\lambda, \nu$ dominant and $\nu \neq \lambda$ the sheaves $R^{k} \Psi_{!} \mathcal{A}_{\lambda}$ are constant on $\mathbb{G}_{a, \overline{\mathbb{F}}_{q}}$.

By Theorem 4.3a of [29] we see that if Conjecture 7.3 holds, then $R^{k} \Psi_{!} \mathcal{A}_{\lambda}=0$ unless $k=2((\lambda, \rho)-1)$. Here we are using the fact that the results of [29], stated there over $\mathbb{C}$, extend to our current context.

7.2. In this subsection we speculate about what could be the analogue of Laumon's sheaf $\mathcal{L}_{E}$ in the case of an arbitrary reductive group. Recall from Sect. 3.2 that $\mathcal{L}_{E}$ is a sheaf on the stack $\operatorname{Coh}_{n}$ canonically attached to a rank $n$ local system $E$ on $X$. This sheaf is used as the starting point of the conjectural construction of the automorphic sheaf on $\mathcal{M}_{n}$ associated to $E$; see [25] and Sect. 3 above.

First we revisit the case of $G L_{n}$ and introduce a scheme $\mathcal{G} r_{X^{(\infty)}}^{+}$with a smooth morphism $q: \mathcal{G} r_{X(\infty)}^{+} \rightarrow \mathcal{C o h}_{n}$, and take the pull-back of $\mathcal{L}_{E}$ to $\mathcal{G} r_{X(\infty)}^{+}$. The scheme $\mathcal{G} r_{X(\infty)}^{+}$classifies pairs $\{L, t\}$, where $L$ is a rank $n$ bundle on $X$ and $t: \mathcal{O}_{X}^{\oplus n} \rightarrow L$ is an embedding of $\mathcal{O}_{X^{-}}$-modules. The scheme $\mathcal{G} r_{X(\infty)}^{+}$is a disjoint union of the smooth schemes $\mathcal{G} r_{X(\infty)}^{+, m}, m \geq 0$, corresponding to bundles of degree $m$. The scheme $\mathcal{G} r_{X(\infty)}^{+, m}$ is isomorphic to the Grothendieck Quot-scheme Quot $_{\mathcal{O}_{X}^{\oplus n} / X / \mathbb{F}_{q}}^{m}$. Recall [13] that Quot $_{\mathcal{O}_{X}^{\oplus n} / X / k}^{m}$ classifies the quotients of $\mathcal{O}_{X}^{\oplus n}$ that are torsion sheaves of length $m$; at the level of points, $\{L, t\}$ corresponds to the quotient of $\mathcal{O}_{X}^{\oplus n}$ by the image of $L^{*}$ under the transpose homomorphism $t^{*}: L^{*} \rightarrow \mathcal{O}_{X}^{\oplus n}$.

The morphism $q: \mathcal{G} r_{X(\infty)}^{+} \rightarrow \operatorname{Coh}_{n}$ sends $\{L, t\}$ to $\mathcal{O}_{X}^{\oplus n} / \operatorname{Im} t^{*}$. In the same way as in Sect. 4.2, one can show that $q$ is smooth. We denote by the same character $\mathcal{L}_{E}$ the pull-back of $\mathcal{L}_{E}$ by $q$. It is the pair $\left(\mathcal{G} r_{X(\infty)}^{+}, \mathcal{L}_{E}\right)$ that we would like to generalize to other groups.

Let us describe the basic structure of $\mathcal{G} r_{X(\infty)}^{+}$. For each $k \geq 1$, we introduce the scheme $\mathcal{G} r_{X^{(k)}}^{+}$over $X^{(k), r s s}$ (see Sect. 3.2), which parametrizes the objects $\left\{\left(x_{1}, \ldots, x_{k}\right), L, t\right\}$, where $\left(x_{1}, \ldots, x_{k}\right)$ is a set of $k$ non-ordered distinct points of $X, L$ is a rank $n$ bundle over $X$, and $t$ is its trivialization over $X-\left\{x_{1}, \ldots, x_{k}\right\}$, which extends to an embedding of $\mathcal{O}_{X}$-modules $\mathcal{O}_{X}^{\oplus n} \rightarrow L$. The fiber of this scheme over $\left(x_{1}, \ldots, x_{k}\right) \in X^{(k), r s s}$ is the product of the $\mathcal{G} r_{x_{i}}^{+}$. It is easy to describe 
the pull-back $\mathcal{L}_{X^{(k)}}^{E,+}$ of $\mathcal{L}_{E}$ under the natural morphism $\delta_{k}: \mathcal{G} r_{X^{(k)}}^{+} \rightarrow \mathcal{G} r_{X^{(\infty)}}^{+}$. In particular, the restriction of $\mathcal{L}_{X^{(k)}}^{E,+}$ to the fiber of $\mathcal{G} r_{X^{(k)}}^{+}$over $\left(x_{1}, \ldots, x_{k}\right)$ is $\bigotimes_{i=1}^{k} \mathcal{L}_{x_{i}}^{E,+}$, where

$$
\mathcal{L}_{x}^{E,+}=\sum_{\lambda \in P_{n}^{++}} \mathcal{A}_{\lambda, x}[|\lambda|(n-1)](|\lambda|(n-1) / 2) \otimes E_{x}(\lambda) .
$$

The set of $\mathbb{F}_{q}$-points of $\mathcal{G} r_{X(\infty)}^{+}$is isomorphic to the quotient $G L_{n}(\mathbb{A})^{+} / G L_{n}(\mathcal{O})$. For groups other than $G L_{n}$ we do not have analogues of the subset $G L_{n}(\mathbb{A})^{+} \subset$ $G L_{n}(\mathbb{A})$, the subset $P_{n}^{++} \subset{ }^{L} P^{+}$, and the subscheme $\mathrm{Gr}^{+}$of the affine Grassmannian. For this reason, we cannot avoid considering a substantially larger object in place of $\mathcal{G} r_{X(\infty)}^{+}$.

Thus, for a reductive group $G$, we consider the set $G(\mathbb{A}) / G(\mathcal{O})$. This set, which we denote by $\operatorname{Gr}(G)_{X(\infty)}$, is isomorphic to the set of isomorphism classes of pairs $\{\mathcal{P}, t\}$, where $\mathcal{P}$ is a principal $G$-bundle over $X$ and $t$ is an isomorphism between $\mathcal{P}$ and the trivial bundle on a Zariski open subset of $X$. It is not difficult to define a functor $\operatorname{Gr}(G)_{X^{(\infty)}}$ from the category of $\mathbb{F}_{q}$-schemes to the category of sets, whose set of $\mathbb{F}_{q}$-points is $\operatorname{Gr}(G)_{X(\infty)}$. It would be desirable to have a notion of perverse sheaf on $\operatorname{Gr}(G)_{X(\infty)}$. A ${ }^{L} G$-local system $E$ on $X$ should give rise to a perverse sheaf $\mathcal{L}_{E}$ on $\mathcal{G r}(G)_{X(\infty)}$, analogous to the sheaf $\mathcal{L}_{E}$ in the case of $G L_{n}$; this sheaf should be irreducible if $E$ is irreducible. Although we do not know how to define such an object, we describe below what its pull-backs should be under certain natural morphisms.

For each $k \geq 1$, following Beilinson and Drinfeld, we introduce the ind-scheme $\mathcal{G r}(G)_{X^{(k)}}$ over $X^{(k), r s s}$, which parametrizes the objects $\left\{\left(x_{1}, \ldots, x_{k}\right), \mathcal{P}, t\right\}$, where $\left(x_{1}, \ldots, x_{k}\right)$ is a set of non-ordered distinct points of $X, \mathcal{P}$ is a principal $G$-bundle over $X$, and $t$ is its isomorphism with the trivial bundle over $X-\left\{x_{1}, \ldots, x_{k}\right\}$. The fiber of this scheme over $\left(x_{1}, \ldots, x_{k}\right) \in X^{(k), r s s}$ is the product of the $\mathcal{G} r_{x_{i}}$ (see [29], Sect. 3). We have an obvious set-theoretic map $\delta_{k}: G r(G)_{X^{(k)}} \rightarrow G(G)_{X^{(\infty)}}$, which can also be defined on the level of functors: schemes $\rightarrow$ sets. The pull-back of $\mathcal{L}_{E}$ to $\mathcal{G r}(G)_{X^{(k)}}$ should be the sheaf $\mathcal{L}_{X^{(k)}}^{E}$ on $\mathcal{G r}(G)_{X^{(k)}}$ (inductive limit of perverse sheaves), such that its restriction to the fiber $\prod_{i=1}^{k} \operatorname{Gr}(G)_{x_{i}}$ over $\left(x_{1}, \ldots, x_{k}\right)$ is $\bigotimes_{i=1}^{k} \mathcal{L}_{x_{i}}^{E}$, where

$$
\mathcal{L}_{x}^{E}=\bigoplus_{\lambda \in{ }^{L} P^{+}} \mathcal{A}_{\lambda, x} \otimes E_{x}(\lambda)^{*}
$$

(up to shifts in degree). Here $E_{x}(\lambda)$ has the same meaning as in the case of $G L_{n}$.

The sheaves $\mathcal{L}_{X^{(k)}}^{E}$ have been previously considered by Beilinson and Drinfeld in the context of the geometric Langlands correspondence. Formula (7.2) is analogous to formula (7.1). The main difference is that in (7.1) the summation is restricted to the subset $P_{n}^{++}$of the set $P_{n}^{+}={ }^{L} P^{+}$of all dominant weights of $G L_{n}$, which does not have an analogue for general $G$ (compare with Sect. 6.4).

Remark 7.1. Let $\mathrm{P}_{\mathcal{G}\left(\mathcal{O}_{x}\right)}\left(\mathcal{G r}(G)_{x}\right)$ be the category of $\mathcal{G}\left(\mathcal{O}_{x}\right)$-equivariant perverse

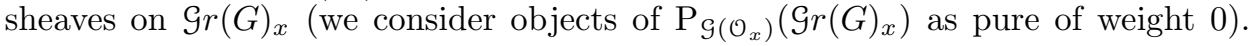
This category is a tensor category, and as such, it is equivalent to the tensor category $\mathcal{R e p}{ }^{L} G$ of finite-dimensional representations of ${ }^{L} G$. To be precise, this result has been proved in [10], [29] over the ground field $\mathbb{C}$ (in this setting, this isomorphism was conjectured by V. Drinfeld; see also [27]). But the proof outlined in [29] can 
be generalized to the $\mathbb{F}_{q}$-case, so here we assume the result to be true over $\mathbb{F}_{q}$ as well.

Note that there is a small error in [29]. The tensor structure (or, more precisely, the commutativity constraint), which is given by the convolution product, should be altered slightly. This alteration does not affect the structure of $\mathrm{P}_{\mathcal{G}\left(\mathcal{O}_{x}\right)}\left(\mathcal{G r}(G)_{x}\right)$ as a monoidal category. We simply replace the perverse sheaf $\mathcal{A}_{\lambda, x}$ with $(-1)^{2(\lambda, \rho)} \mathcal{A}_{\lambda, x}$, where the sign $(-1)^{2(\lambda, \rho)}$ is to be viewed as a formal symbol. The symbol $(-1)^{2(\lambda, \rho)}$ has the effect of making the cohomology of $(-1)^{2(\lambda, \rho)} \mathcal{A}_{\lambda, x}$ lie in even degrees only. Then the equivalence of categories $\operatorname{Rep}^{L} G \rightarrow \mathrm{P}_{\mathcal{G}_{\left(\mathcal{O}_{x}\right)}}\left(\operatorname{Gr} r(G)_{x}\right)$ takes the irreducible representation $V(\lambda)$ to the perverse sheaf $(-1)^{2(\lambda, \rho)} \mathcal{A}_{\lambda, x}$. With this adjustment the sign in Proposition 5.1 disappears and the equivalence above can be viewed as a categorical version of the Satake isomorphism $\operatorname{Rep}^{L} G \rightarrow \mathcal{H}$ (see Theorem 5.1). Indeed, an equivalence of two categories induces an isomorphism of their Grothendieck rings. But the Grothendieck ring of $\mathrm{P}_{\mathcal{G}\left(\mathcal{O}_{x}\right)}\left(\mathcal{G} r(G)_{x}\right)$ is canonically isomorphic to the Hecke algebra $\mathcal{H}$ via the "faisceaux-fonctions" correspondence.

Now consider the left regular representation of ${ }^{L} G$,

$$
\bigoplus_{\lambda \in{ }^{L} P^{+}} V(\lambda) \otimes V(\lambda)^{*}
$$

as an ind-object of the category $\operatorname{Rep}^{L} G$. The corresponding ind-object of the category $\mathrm{P}_{\mathcal{G}\left(\mathcal{O}_{x}\right)}\left(\mathcal{G r}(G)_{x}\right)$ is $\mathcal{L}_{x}^{E}$ given by formula (7.2), adjusted for the formal signs, i.e.,

$$
\mathcal{L}_{x}^{E}=\bigoplus_{\lambda \in{ }^{L} P^{+}}(-1)^{2(\lambda, \rho)} \mathcal{A}_{\lambda, x} \otimes E_{x}(\lambda)^{*}
$$

Remark 7.2. Let $L_{X}^{E}$ be the function associated to the sheaf $\mathcal{L}_{X}^{E}$, and let $L_{x}^{E}$ be the restriction of $L_{X}^{E}$ to $G r(G)_{x} \subset G r(G)_{X}$. Using Proposition 5.1 we obtain:

$$
L_{x}^{E}=\sum_{\lambda \in{ }^{L} P^{+}} \operatorname{Tr}\left(\gamma_{x}, V(\lambda)^{*}\right) \cdot H_{\lambda, x},
$$

where $\gamma_{x}=\sigma\left(\operatorname{Fr}_{x}\right)$. Hence the function $L_{x}^{E}$ coincides with the function $L_{\gamma_{x}^{-1}}$ given by formula (6.12). According to Proposition 6.2, the series (7.4) converges absolutely if $q_{x}^{-1}<\left|\alpha\left(\gamma_{x}\right)\right|<q_{x}, \forall \alpha \in \Delta_{+}$, and is proportional to the spherical function $S_{\gamma_{x}^{-1}}$.

\section{ACKNOWLEDGMENTS}

We express our gratitude to J. Bernstein and I. Mirković for valuable discussions and to B. Gross for useful remarks concerning the Whittaker models. We thank A. Beilinson and V. Drinfeld for sharing with us their ideas and unpublished results about the affine Grassmannian, which we used in Sect. 7.2.

We are indebted to the referee for valuable comments and suggestions.

The research of E. Frenkel was supported by grants from the Packard and Sloan Foundations, and by NSF grants DMS 9501414 and DMS 9304580. The research of D. Gaitsgory was supported by NSF grant DMS 9304580. D. Kazhdan was supported by NSF grant DMS 9622742. K. Vilonen was supported by NSF grant DMS 9504299 and by NSA grant MDA 90495H103. 


\section{REFERENCES}

[1] A. Beauville, Y. Laszlo, Conformal blocks and generalized theta functions, Comm. Math. Phys. 164 (1994) 385-419. MR 95k:1401

[2] A. Beauville, Y. Laszlo, Un lemme de descente, C.R. Acad. Sci. Paris, Sér. I Math. 320 (1995) 335-340. MR 96a:14049

[3] J. Bernstein, A. Zelevinsky, Representations of the group $G L(n, F)$ where $F$ is a local nonArchimedean field, Russ. Math. Surv. 31 (1976) 1-68.

[4] R.K. Brylinski, Limits of weight spaces, Lusztig's q-analogs, and fiberings of adjoint orbits, Journal of AMS 2 (1989) 517-533. MR 90g:17011

[5] W. Casselman, J. Shalika, The unramified principal series of p-adic groups II. The Whittaker function, Comp. Math. 41 (1980) 207-231. MR 83i:22027

[6] P. Deligne, Le conjecture de Weil II, Publ. IHES 52 (1980) 137-252 MR 83c:14017

[7] V.G. Drinfeld, Two-dimensional $\ell$-adic representations of the fundamental group of a curve over a finite field and automorphic forms on GL(2), Amer. J. Math. 105 (1983) 85-114. MR 84i: 12011

[8] V.G. Drinfeld, C. Simpson, B-structures on G-bundles and local triviality, Math. Res. Lett. 2 (1995) 823-829. MR 96k:14013

[9] I.M. Gelfand, D. Kazhdan, Representations of the group $G L(n, K)$ where $K$ is a local field, in Lie Groups and Their Representations, ed. I.M. Gelfand, pp. 95-118, Adam Hilder Publ., 1975. MR 53:8334

[10] V. Ginzburg, Perverse sheaves on a loop group and Langlands duality, Preprint alggeom/9511007 (1995).

[11] B.H. Gross, On the Satake isomorphism, Preprint (1996).

[12] B.H. Gross, D. Prasad, On the decomposition of a representation of $S_{n} O_{n}$ when restricted to $S O_{n-1}$, Canadian J. Math. 44 (1992) 974-1002. MR 93j:22031

[13] A. Grothendieck, Techniques de construction et théorèmes d'existence en géométrie algébrique IV: les schémas de Hilbert, Séminaire Bourbaki 221 (1960/61), Benjamin (1966).

[14] W.H. Hesselink, Characters of the nullcone, Math. Ann. 252 (1980) 179-182. MR 82c:1700

[15] H. Jacquet, R.P. Langlands, Automorphic Forms on $G L(2)$, Lect. Notes in Math. 114, Springer Verlag, 1970. MR 53:5481

[16] H. Jacquet, I.I. Piatetski-Shapiro, J. Shalika, Automorphic forms on GL(3), I and II, Ann. Math. 109 (1979) 169-258. MR 80i:10034a; MR 80i:10034b

[17] S. Kato, Spherical functions and a q-analogue of Kostant's weight multiplicity formula, Inv. Math. 66 (1982) 461-468. MR 84b:22030

[18] B. Kostant, Lie group representations on polynomial rings, Amer. J. Math. 85 (1963) 327404. MR 28:1252

[19] R.P. Langlands, Problems in the theory of automorphic forms, in Lect. Notes in Math. 170, pp. 18-61, Springer Verlag, 1970. MR 46:1758

[20] Y. Laszlo, Ch. Sorger, The line bundles on the moduli of parabolic G-bundles over curves and their sections, Ann. Sci. École Norm. Sup. 30 (1997) 499-525. CMP 97:14

[21] G. Laumon, Transformation de Fourier, constants d'équations fonctionnelles et conjecture de Weil, Publ. IHES 65 (1987) 131-210. MR 88g:14019

[22] G. Laumon, Correspondance de Langlands géométrique pour les corps de fonctions, Duke Math. J. 54 (1987) 309-359. MR 88g:11086

[23] G. Laumon, Faisceaux automorphes liés aux séries d'Eisenstein, in Automorphic Forms, Shimura varieties, and $L$-functions, vol. 1, pp. 227-281, Academic Press, 1990. MR 91k:11106

[24] G. Laumon, L. Moret-Bailly, Champs algébriques, Preprint 92-42, Université Paris Sud, 1992.

[25] G. Laumon, Faisceaux automorphes pour $G L_{n}$ : la première construction de Drinfeld, Preprint alg-geom/9511004 (1995).

[26] G. Lusztig, Green polynomials and singularities of unipotent classes, Adv. Math. 42 (1981) 169-178. MR 83c:20059

[27] G. Lusztig, Singularities, character formulas, and a q-analogue of weight multiplicities, Astérisque 101 (1983) 208-229. MR 85m:17005

[28] I.G. Macdonald, Spherical Functions on a Group of $p$-adic Type, Ramanujan Inst. Publ., University of Madras, India, 1971. MR 55:8261

[29] I. Mirković, K. Vilonen Perverse sheaves on loop Grassmannians and Langlands duality, Preprint alg-geom/9703010, to appear in the proceedings of a conference on the geometric Langlands correspondence at Luminy, June 1995. 
[30] I.I. Piatetski-Shapiro, Euler subgroups, in Lie Groups and Their Representations, ed. I.M. Gelfand, pp. 597-620, Adam Hilder Publ., 1975. MR 53:10720

[31] I. Satake, Theory of spherical functions on reductive algebraic groups over p-adic fields, Publ. IHES 18 (1963) 1-69. MR 33:4059

[32] J.A. Shalika, The multiplicity one theorem for $G L_{n}$, Ann. Math. 100 (1974) 171-193. MR 50:545

[33] T. Shintani, On an explicit formula for class 1 Whittaker functions on $G L_{n}$ over $\mathfrak{P}$-adic fields, Proc. Japan Acad. 52 (1976) 180-182. MR 53:10991

[34] A. Weil, Dirichlet Series and Automorphic Forms, Lect. Notes in Math. 189, Springer Verlag, 1971.

(E. Frenkel and D. Kazhdan) Department of Mathematics, Harvard University, CamBRIDGe, Massachusetts 02138

(D. Gaitsgory) School of Mathematics, Institute for Advanced Study, Princeton, New JERSEY 08540

(K. Vilonen) Department of Mathematics, Brandeis University, Waltham, MassachuSETTS 02254 